This item was submitted to Loughborough's Research Repository by the author.

Items in Figshare are protected by copyright, with all rights reserved, unless otherwise indicated.

\title{
Vanishing characteristic speeds and critical dispersive points in nonlinear interfacial wave problems
}

PLEASE CITE THE PUBLISHED VERSION

https://doi.org/10.1063/1.4998803

PUBLISHER

AIP Publishing () The Author

VERSION

AM (Accepted Manuscript)

LICENCE

CC BY-NC-ND 4.0

\section{REPOSITORY RECORD}

Ratliff, Daniel J.. 2019. "Vanishing Characteristic Speeds and Critical Dispersive Points in Nonlinear Interfacial Wave Problems". figshare. https://hdl.handle.net/2134/35903. 


\title{
Vanishing Characteristic Speeds and Critical Dispersive Points in Nonlinear Interfacial Wave Problems
}

\author{
Daniel J. Ratliff
}

November 8, 2018

\begin{abstract}
Criticality plays a central role in the study of reductions and stability of hydrodynamical systems. At critical points, it is often the case that nonlinear reductions with dispersion arise to govern solution behaviour. By considering when such models become bidirectional and lose their initial dispersive properties, it will be shown that higher order dispersive models may be supported in hydrodynamical systems. Precisely, this equation is a two-way Boussinesq equation with sixth order dispersion. The case of two layered shallow water is considered to illustrate this, and it is reasoned why such an environment is natural for such a system to emerge. Further, it is demonstrated that the regions in parameter space for nontrivial flows admit such reductions is vast and forms a continuum. The reduced model is then numerically simulated to illustrate how the two-way and higher dispersive properties suggest more exotic families of solitary wave solutions can emerge in stratified flows.
\end{abstract}

\section{Introduction}

Stratified flows are a rich source of dynamical behaviours and nonlinear reductions. This is in no small part to the large parameter space these systems offer, and many well-known reductions have been shown to emerge from two (for example, $[11,12,15,20]$ ) and threelayered $([16,17])$ shallow water flows. On the whole these systems are nondegenerate, but it can be shown (e.g. [27]) that there are entire continuums of points where coefficients within these systems vanish and other balances must be sought. This paper deals with one such case for two layered flows, where the Korteweg-de Vries (KdV) equation degenerates in both of its linear terms and results in a two-way highly dispersive equation. This equation, referred to herein as the the higher order two-way Boussinesq equation, takes the form

$$
c_{0} A_{T T}+\left(\frac{1}{2} c_{1} A^{2}+c_{2} A_{X T}+c_{3} A_{X X X X}\right)_{X X}=0,
$$

for constants $c_{i}$. A similar equation to the above has previously appeared in the literature [13], however the key aim of this paper is to present a new derivation of (1) from first principles, which highlights how the system emerges due to a codimension three singularity (in the sense that three conditions are required to be met in order for it to emerge). Through 
this identification of the relation between the singularity theory and the emergence of (1), such an equation may be readily derived in other contexts where analogous conditions are met.

The literature on two-layer flows has already connected the degeneracy of conservation laws to the emergence of instability and nonlinear phenomenon [3, 8, 22], and in other contexts the analysis of multiple conservation laws in provides valuable insight into changes in stability and thus system behaviour [7,21]. This will be the primary framework in which we discuss and derive the reduction presented in this paper, relating its emergence to properties of the conservation laws within the stratified flow and use them to ultimately generate some of the coefficients in the result.

Arising from perturbation analyses at such stability boundaries is the KdV equation,

$$
a_{0} A_{T}+a_{1} A A_{X}+a_{2} A_{X X X}=0
$$

for unknown function $A(X, T)$, nonzero coefficients $a_{i}$ and slow variables $X, T$. These are typically functions of parameters appearing in the original system (such as the densities, velocities and thicknesses of each flow), and so vary depending on the state of the full problem. In cases where varying these parameters causes the coefficient of the time derivative term to vanish, a different balance of terms in the reduction must be sought and instead the two-way Boussinesq,

$$
c_{0} A_{T T}+\left(\frac{1}{2} a_{1} A^{2}+a_{2} A_{X X}\right)_{X X}=0,
$$

appears. The new coefficient $c_{0}$ is again a function of physical parameters. The emergence of this equation in two layered flow was shown to arise within a large subset of the parameter space in [27]. The aim of this paper is to take this idea of rebalance one stage further, and to show that when the dispersive coefficient $a_{2}$ also vanishes the appropriate nonlinear reduction becomes (1). Thus the higher order two-way Boussinesq can be seen as the leading order correction to the $\mathrm{KdV}$ equation when both of its linear terms vanish. The new balance of dispersion morphs to incorporate higher dispersive effects as well as a mixed dispersive element. In essence, this is simply a bidirectional fifth order KdV - almost - since the mixed derivative term changes sign depending on the direction of propagation chosen.

The natural way to undertake such an approach is through modulation [4, 25, 26]. This technique makes use of the symmetries inherent to the problem to construct the relevant reductions from derivatives of the conservation law components. In addition, the criteria for emergence are formulated from these and so the problem may be diagnosed entirely from the basic state (that is, the solution which these equations are derived about). The approach has already demonstrated how both the KdV and two-way Boussinesq may arise from twolayered flow $[26,27]$, and the novelty of this paper takes it one step further by considering degeneration of the dispersive component of these equations.

The advantages of the modulation approach numerous. Firstly, it allows one to relate a number of the coefficients of the resulting nonlinear PDE to the conservation laws the system possesses, and so their computation may be done in advance. Secondly, the number of unknown functions introduced in the asymptotics is reduced, which in this setting reduces the number of unknowns initially dealt with from four (two for the velocities and two for each free surface) into a family generated from two unknowns. In fact, in nonlinear regimes 
this is further reduced to a single family with a scalar unknown. Additionally, due to the way the ansatz will be constructed, several unimportant terms automatically vanish due to properties of the uniform flow solution which simplifies the analysis. Finally, although the focus will be on a stratified shallow water system, the way that the modulation approach will be formulated here will allow the results to be applicable to additional systems providing all the relevant conditions can be met. It is for these reasons the modulation approach is adopted instead of alternate methodologies to obtain the equation (27).

In the scope of modulation, dispersion arises from a Jordan chain analysis. The shallow water system typically admits chains of length two, which become length four when the first characteristic speed of the shallow water system vanishes [26]. This chain has the potential to be extended even further due to the large parameter space of the two layered system, and this is explored within this paper and shown to generate higher order dispersion. This also has the natural consequence that a mixed dispersive term emerges, generated by combining chains relating to both space and time.

The stratified shallow water system is a natural environment to derive the system (1), since such an equation would be codimension three and there are many parameters one can vary in such a system. This is explored within the paper, and the existence of points where all three conditions can be met physically is demonstrated. It will be shown that there are entire continuums of points where (1) emerges as the leading order nonlinear PDE.

An additional contribution made in this paper is the derivation of such an equation for finite velocity flows in a stationary frame. Typically, such nonlinear reductions are obtained for stationary flows in each layer with a travelling wave co-ordinate whose wavespeed is suitably chosen (see, for example, [15] and [20]). This current work generalises the approach taken in these to consider scenarios where the background flow is nonzero, and in doing so the velocities of the fluids appear within the conditions for the equation (1) to emerge. Undertaking the asymptotics in a fixed frame instead of a moving co-ordinate will mean that the velocities and other system parameters of each flow are suitably chosen instead in order to satisfy certain constraints, which will discussed within the manuscript.

The format of this paper is natural. We begin with the discussion of the governing equations in $\S 2$, including their multisymplectification, the relevant conservation laws for this system and their linearisation about constant velocity states. The criticality is discussed in $\S 3$, and it is demonstrated that the relevant conditions for the model to emerge can not only be met, but there are a large set of parameters for which is is realisable. The reduction is undertaken in $\S 4$, demonstrating how the modulation approach leads to the nonlinear system of interest. This system is then studied in $\S 5$, which also presents numerical simulations that lead to solitary travelling waves of exotic forms. Concluding remarks then appear in $\S 6$.

\section{Governing Equations and Abstract Set-Up}

The physical system considered here is a two-layered shallow fluid system restricted to $1+1$ dimensions. In particular we assume each fluid has constant density, is inviscid, irrotational and incompressible. The fluid bed lies at $y=0$ with the interface between the two fluids being described by the graph $y=\eta(x, t)$ and the upper-most free surface by $y=\chi(x, t)$. For simplicity, we neglect surface tension effects and thus use the model proposed by BAINES [1] 
but we also incorporate dispersion terms into the model through the results of DonALDson [14]. The model equations are thus:

$$
\begin{gathered}
\rho_{1}\left(\eta_{t}+\left(\eta u_{1}\right)_{x}\right)=0, \\
\rho_{2}\left(\chi_{t}+\left(\chi u_{2}\right)_{x}\right)=0, \\
\rho_{1}\left(u_{1}\right)_{t}+\frac{\rho_{1}}{2}\left(u_{1}^{2}\right)_{x}+g \rho_{1} \eta_{x}+g \rho_{2} \chi_{x}=a_{11} \eta_{x x x}+a_{12} \chi_{x x x}, \\
\rho_{2}\left(u_{2}\right)_{t}+\frac{\rho_{2}}{2}\left(u_{2}^{2}\right)_{x}+g \rho_{2} \eta_{x}+g \rho_{2} \chi_{x}=a_{21} \eta_{x x x}+a_{22} \chi_{x x x} .
\end{gathered}
$$

In the above we take $u_{i}, \rho_{i}$ are the velocity and density in layer $i$. Layer two resides above layer one, and so for stable stratification we require $r=\frac{\rho_{2}}{\rho_{1}}<1$. The dispersion constants $a_{i j}$ are given by

$$
\begin{aligned}
& a_{11}=-\frac{1}{3} \rho_{1} g \eta_{0}^{2}-\rho_{2} g \eta_{0} \chi_{0}-\frac{1}{2} \rho_{2} g \chi_{0}^{2} \\
& a_{12}=a_{21}=-\frac{1}{6} \rho_{2} g \eta_{0}^{2}-\frac{1}{4} \rho_{2} g \eta_{0} \chi_{0}-\frac{1}{2} \rho_{2} g r \eta_{0} \chi_{0}-\frac{5}{12} \rho_{2} g \chi_{0}^{2} \\
& a_{22}=-\frac{1}{2} \rho_{2} g r \eta_{0} \chi_{0}-\frac{1}{3} \rho_{2} g \chi_{0}^{2}
\end{aligned}
$$

This setup is presented in figure 1 .

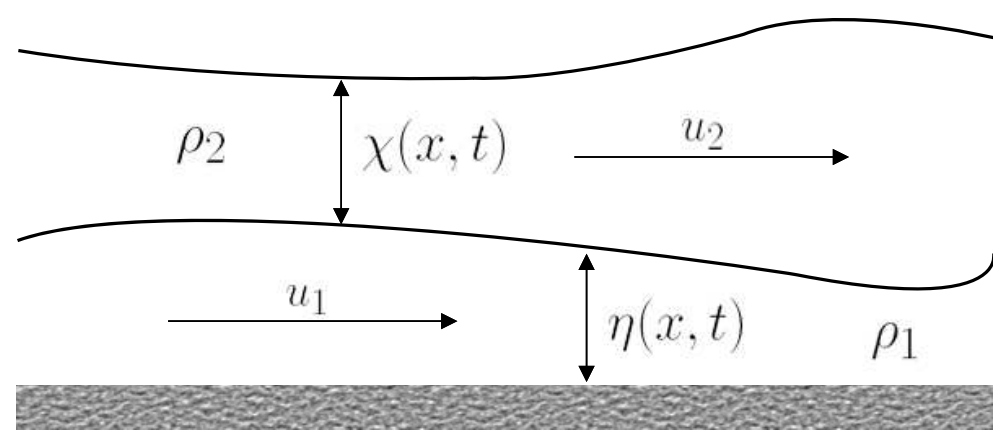

Figure 1: A diagram of the system governed by (2).

Due to the irrotationality of the flows, we may introduce the velocity potentials for each fluid as $\phi_{i}$. This allows us to integrate the latter two equations of (2) to form the potential system

$$
\begin{aligned}
\rho_{1}\left(\eta_{t}+\left(\eta\left(\phi_{1}\right)_{x}\right)_{x}\right) & =0 \\
\rho_{2}\left(\chi_{t}+\left(\chi\left(\phi_{2}\right)_{x}\right)_{x}\right) & =0 \\
\rho_{1}\left(\phi_{1}\right)_{t}+\frac{\rho_{1}}{2}\left(\phi_{1}\right)_{x}^{2}+g \rho_{1} \eta+g \rho_{2} \chi & =R_{1}+a_{11} \eta_{x x}+a_{12} \chi_{x x}, \\
\rho_{2}\left(\phi_{2}\right)_{t}+\frac{\rho_{2}}{2}\left(\phi_{2}\right)_{x}^{2}+g \rho_{2} \eta+g \rho_{2} \chi & =R_{2}+a_{21} \eta_{x x}+a_{22} \chi_{x x},
\end{aligned}
$$


where $R_{i}$ are the Bernoulli constants for each layer. The first two equations of this system (3a) and (3b) form the conservation laws for this system, corresponding to the conservation of mass in each layer.

Of interest in this paper is the constant velocity solution in each layer, associated with the affine symmetry of the velocity potentials arising from (3. In light of this, we choose to take

$$
\phi_{i}=u_{i} x+\omega_{i} t+\theta_{0} \equiv \theta_{i},
$$

for $u_{i}, \omega_{i}, \theta_{0}$ constants. In particular, the $u_{i}$ are the velocities in each flow. From this one may find the layer thicknesses as

$$
\begin{aligned}
& \eta=\eta_{0}=\frac{1}{g\left(\rho_{1}-\rho_{2}\right)}\left(\frac{1}{2}\left(\rho_{2} u_{2}^{2}-\rho_{1} u_{1}^{2}\right)+R_{1}-R_{2}+\rho_{2} \omega_{2}-\rho_{1} \omega_{1}\right), \\
& \chi=\chi_{0}=\frac{\rho_{1}}{g\left(\rho_{1}-\rho_{2}\right)}\left(R_{2}-R_{1}+\frac{1}{2}\left(u_{1}^{2}-u_{2}^{2}\right)+\omega_{1}-\omega_{2}\right) .
\end{aligned}
$$

\subsection{Transformation to Multisymplectic Formulation and Conser- vation Laws}

The Lagrangian that generates the system (3) through variations is given by

$$
\begin{aligned}
\mathscr{L}=\iint \mathcal{L}\left(\phi_{1}, \phi_{2}, \eta, \chi,\left(\phi_{1}\right)_{x},\left(\phi_{2}\right)_{x}, \eta_{x}, \chi_{x}\right) d x d t \\
=\iint\left[\rho_{1}\left(\eta\left(\phi_{1}\right)_{t}+\frac{1}{2} \eta\left(\phi_{1}\right)_{x}^{2}+\frac{g}{2} \eta^{2}\right)+\frac{a_{11}}{2} \eta_{x}^{2}+a_{12} \eta_{x} \chi_{x}+\frac{a_{22}}{2} \chi_{x}^{2}\right. \\
\left.\quad+\rho_{2}\left(\chi\left(\phi_{2}\right)_{t}+\frac{1}{2} \chi\left(\phi_{2}\right)_{x}^{2}+g \eta \chi+\frac{g}{2} \chi^{2}\right)-R_{1} \eta-R_{2} \chi\right] d x d t .
\end{aligned}
$$

One way to obtain this Lagrangian is to consider the addition of two independent single layer shallow water Lagrangians (like that which appears in [4]) and include the relevant coupling terms.. The idea is to replace the terms depending on derivatives through the use of Legendre transformations. Define the following:

$$
\begin{array}{r}
p_{1}=\frac{\partial \mathcal{L}}{\partial\left(\phi_{1}\right)_{x}}=\rho_{1} \eta\left(\phi_{1}\right)_{x}, \quad p_{2}=\frac{\partial \mathcal{L}}{\partial\left(\phi_{2}\right)_{x}}=\rho_{2} \eta\left(\phi_{2}\right)_{x} \\
\sigma=\frac{\partial \mathcal{L}}{\partial \eta_{x}}=a_{11} \eta_{x}+a_{12} \chi_{x}, \quad \tau=\frac{\partial \mathcal{L}}{\partial \chi_{x}}=a_{12} \eta_{x}+a_{22} \chi_{x}
\end{array}
$$

which then allows one to write (4) as

$$
\begin{aligned}
\mathscr{L}=\iint & {\left[\rho_{1} \eta\left(\phi_{1}\right)_{t}+\frac{p_{1}^{2}}{2 \rho_{1} \eta}+\frac{g \rho_{1}}{2} \eta^{2}+\frac{1}{2} \Gamma_{1} \sigma^{2}+\Gamma_{2} \tau \sigma+\frac{1}{2} \Gamma_{3} \tau^{2}\right.} \\
& \left.+\rho_{2} \chi\left(\phi_{2}\right)_{t}+\frac{p_{2}^{2}}{2 \rho_{2} \chi}+g \rho_{2} \eta \chi+\frac{g \rho_{2}}{2} \chi^{2}-R_{1} \eta-R_{2} \chi\right] d x d t
\end{aligned}
$$

where the constants $\Gamma_{i}$ are given by

$$
\Gamma_{1}=\frac{a_{11}}{a_{11} a_{22}-a_{12}^{2}}, \quad \Gamma_{2}=-\frac{a_{12}}{a_{11} a_{22}-a_{12}^{2}}, \quad \Gamma_{3}=\frac{a_{22}}{a_{11} a_{22}-a_{12}^{2}} .
$$


Finally, we introduce the state vector $Z=\left(\phi_{1}, \eta, \phi_{2}, \chi, p_{1}, \sigma, p_{2}, \tau\right)^{T}$ to allow us to write the system in multisymplectic form:

$$
\mathscr{L}=\iint\left[\frac{1}{2}\left\langle Z, \mathbf{M} Z_{t}\right\rangle+\frac{1}{2}\left\langle Z, \mathbf{J} Z_{x}\right\rangle-S(Z)\right] d x d t,
$$

where $\langle\cdot, \cdot\rangle$ is the standard inner product on $\mathbb{R}^{n}$. The skew symmetric matrices $\mathbf{M}, \mathbf{J}$ in the above are given by

$$
\mathbf{M}=\left(\begin{array}{cccc}
\rho_{1} \mathbf{j} & \mathbf{0}_{2} & \mathbf{0}_{2} & \mathbf{0}_{2} \\
\mathbf{0}_{2} & \rho_{2} \mathbf{j} & \mathbf{0}_{2} & \mathbf{0}_{2} \\
\mathbf{0}_{2} & \mathbf{0}_{2} & \mathbf{0}_{2} & \mathbf{0}_{2} \\
\mathbf{0}_{2} & \mathbf{0}_{2} & \mathbf{0}_{2} & \mathbf{0}_{2}
\end{array}\right), \quad \mathbf{j}=\left(\begin{array}{cc}
0 & -1 \\
1 & 0
\end{array}\right), \quad \mathbf{J}=\left(\begin{array}{cc}
\mathbf{0}_{4} & -\mathbf{I}_{4} \\
\mathbf{I}_{4} & \mathbf{0}_{4}
\end{array}\right)
$$

and the function $S$ is defined as

$$
S(Z)=R_{1} \eta+R_{2} \chi-\frac{\rho_{1}}{2} \eta^{2}-\rho_{2} \eta \chi-\frac{\rho_{2}}{2} \chi^{2}+\frac{1}{2} \Gamma_{1} \sigma^{2}+\Gamma_{2} \sigma \tau+\frac{1}{2} \Gamma_{3} \tau^{2}+\frac{p_{1}^{2}}{2 \rho_{1} \eta}+\frac{p_{2}^{2}}{2 \rho_{2} \chi}
$$

In this form, one may write the Euler-Lagrange equations as

$$
\mathbf{M} Z_{t}+\mathbf{J} Z_{x}=\nabla S(Z) .
$$

The first four equations of this recover (3), whereas the last four recover the Legendre transform results (5). One may then write the constant velocity solution as

$$
Z=\widehat{Z}(\boldsymbol{\theta}, \mathbf{u}, \boldsymbol{\omega})=\left(\begin{array}{c}
\theta_{1} \\
\eta_{0} \\
\theta_{2} \\
\chi_{0} \\
\rho_{1} u_{1} \eta_{0} \\
0 \\
\rho_{2} u_{2} \chi_{0} \\
0
\end{array}\right), \quad \boldsymbol{\theta}=\left(\begin{array}{c}
\theta_{1} \\
\theta_{2}
\end{array}\right), \quad \mathbf{u}=\left(\begin{array}{l}
u_{1} \\
u_{2}
\end{array}\right), \quad \boldsymbol{\omega}=\left(\begin{array}{c}
\omega_{1} \\
\omega_{2}
\end{array}\right)
$$

which satisfies the PDE

$$
\sum_{i=1}^{2}\left(\left(\omega_{i} \mathbf{M}+u_{i} \mathbf{J}\right) \widehat{Z}_{\theta_{i}}\right)=\nabla S(\widehat{Z}) .
$$

An important component of the asymptotics is the linear operator about $\widehat{Z}$, which can be found as

$$
\mathbf{L}=\mathrm{D}^{2} S(\widehat{Z})-\sum_{i=1}^{2}\left(\omega_{i} \mathbf{M}+u_{i} \mathbf{J}\right) \partial_{\theta_{i}}
$$

and by differentiating (7) with respect to $\theta_{i}, u_{i}$ and $\omega_{i}$ one finds the following results:

$$
\mathbf{L} \widehat{Z}_{\theta_{i}}=0, \quad \mathbf{L} \widehat{Z}_{u_{i}}=\mathbf{J} \widehat{Z}_{\theta_{i}}, \quad \mathbf{L} \widehat{Z}_{\omega_{i}}=\mathbf{M} \widehat{Z}_{\theta_{i}} .
$$


The first result in the above highlights that the kernel has at least dimension 2, and for the purposes of this paper we assume it is no larger. As such, this leads to the solvability condition for inhomogenous systems involving the operator $\mathbf{L}$ as

$$
\mathbf{L} Q=R \quad \text { is solvable if }\left\langle\widehat{Z}_{\theta_{i}}, R\right\rangle=0, \quad i=1,2 .
$$

The latter two results in (9) highlight the presence of Jordan chain structures, of which only the second result is important. A discussion on the relevant Jordan chain theory and its role in the asymptotics appears in $\S 2.3$.

\subsection{Conservation Laws and Criticality}

Now that the system is in multisymplectic form, define the following vectors:

$$
\mathbf{A}(\mathbf{u}, \boldsymbol{\omega})=\left(\begin{array}{c}
\left\langle\widehat{Z}, \mathbf{M} \widehat{Z}_{\theta_{1}}\right\rangle \\
\left\langle\widehat{Z}, \mathbf{M} \widehat{Z}_{\theta_{2}}\right\rangle
\end{array}\right)=\left(\begin{array}{c}
\rho_{1} \eta_{0} \\
\rho_{2} \chi_{0}
\end{array}\right), \quad \mathbf{B}(\mathbf{u}, \boldsymbol{\omega})=\left(\begin{array}{c}
\left\langle\widehat{Z}, \mathbf{J} \widehat{Z}_{\theta_{1}}\right\rangle \\
\left\langle\widehat{Z}, \mathbf{J} \widehat{Z}_{\theta_{2}}\right\rangle
\end{array}\right)=\left(\begin{array}{c}
\rho_{1} u_{1} \eta_{0} \\
\rho_{2} u_{2} \chi_{0}
\end{array}\right) .
$$

These are obtained by evaluating the system's conservation laws (3a) and (3b) along the uniform flow solution. In particular, the modulation theory will see the Jacobians and Hessians of the above. Of these, the one of primary importance is that of $\mathbf{B}$ with respect to $\mathbf{u}, \mathrm{D}_{\mathbf{u}} \mathbf{B}$. Its role in the reduction is that is forms the primary condition for nonlinearity to emerge in the final PDE, which happens precisely when it possesses a zero eigenvalue. Therefore one of our principle assumptions in this paper is

$$
\operatorname{det}\left[D_{\mathbf{u}} \mathbf{B}\right]=0 \quad \text { so that } \quad \mathrm{D}_{\mathbf{u}} \mathbf{B} \boldsymbol{\zeta}=\mathbf{0} .
$$

For this system, this condition reduces to

$$
\left(1-F_{1}^{2}\right)\left(1-F_{2}^{2}\right)=r,
$$

where we have introduced the Froude numbers in each layer

$$
F_{1}^{2}=\frac{u_{1}^{2}}{g \eta_{0}}, \quad F_{2}^{2}=\frac{u_{2}^{2}}{g \chi_{0}} .
$$

Interestingly, this condition has been shown to correspond to when first characteristic speed for the system (2) vanishes [22]. The change in sign of this speed forms the threshold for a change of stability within the flow, often leading to the generation of hydraulic jumps [3]. When this zero determinant condition is met, one may readily find the eigenvector $\boldsymbol{\zeta}$ as

$$
\boldsymbol{\zeta}=\left(\begin{array}{l}
\zeta_{1} \\
\zeta_{2}
\end{array}\right)=\left(\begin{array}{c}
\rho_{2} u_{1} u_{2} \\
g \rho_{1} \eta_{0}\left(1-r-F_{1}^{2}\right)
\end{array}\right)
$$

Physically, $\boldsymbol{\zeta}$ denotes the proportionality between the two velocity perturbations for which the above criticality is satisfied, which will ultimately lead to the emergence of a nonlinear scalar PDE from the analysis. 
Within the setting of this paper, we consider the degeneracy of the second characteristic speed as well, which gives the condition

$$
\frac{u_{1}}{g \eta_{0}}\left(1-F_{2}^{2}\right)+\frac{u_{2}}{g \chi_{0}}\left(1-F_{1}^{2}\right)=0
$$

This condition arises in this setting from the projection

$$
\boldsymbol{\zeta}^{T}\left(\mathrm{D}_{\mathbf{u}} \mathbf{A}+\mathrm{D}_{\boldsymbol{\omega}} \mathbf{B}\right) \boldsymbol{\zeta}=0
$$

which can be shown to both relate to the vanishing of the time derivative term in the $\mathrm{KdV}$ [26], as well as the condition for the matrix

$$
\left(\begin{array}{cc}
\mathbf{0}_{2} & -\mathrm{D}_{\boldsymbol{\omega}} \mathbf{A} \\
\mathrm{D}_{\mathbf{u}} \mathbf{B} & \mathrm{D}_{\mathbf{u}} \mathbf{A}+\mathrm{D}_{\boldsymbol{\omega}} \mathbf{B}
\end{array}\right)
$$

having a zero eigenvalue of geometric multiplicity 2 [27]. In this case one is able to define $\gamma$, which can be thought of as a generalised eigenvector, with the property

$$
\left(\mathrm{D}_{\mathbf{u}} \mathbf{A}+\mathrm{D}_{\boldsymbol{\omega}} \mathbf{B}\right) \boldsymbol{\zeta}=\mathrm{D}_{\mathbf{u}} \mathbf{B} \boldsymbol{\gamma}
$$

One may then find $\gamma$ as

$$
\gamma=\left(\begin{array}{l}
\gamma_{1} \\
\gamma_{2}
\end{array}\right)=\frac{\rho_{2}}{(1-r)}\left[\frac{2 F_{1}^{2} u_{2}}{1-r-F_{1}^{2}}+u_{1}+u_{2}\right]\left(\begin{array}{l}
1 \\
0
\end{array}\right)
$$

up to shifts in $\boldsymbol{\zeta}$. When both the criterion (14) and (11) are met, a second order time derivative appears in the resulting nonlinear PDE and thus two-way waves become applicable. Moreover the vectors discussed here, along with an additional vector arising from the degeneracy of the dispersion, will appear within the modulation construction explicitly in order to simplify the analysis. The fact that these conditions are assumed to be satisfied a priori facilitates the need for only one unknown function $U$ within the ansatz itself and reduces the complexity of the reduction.

\subsection{Linearisation of the Basic State and Jordan Chain Analysis}

The other important features one obtains via the multisymplectic reformulation are Jordan chain structures. In this paper, two types emerge and relate to different dispersive terms. The first is a more classical form and is reminiscent of that previously seen in this setting [26], but a longer chain is now considered. The other is formed from the coalescence of two independent chains.

\subsubsection{Primary Chain Analysis}

The primary Jordan chains appearing within this paper initially takes the form

$$
\mathbf{L} \xi_{1}^{j}=0, \quad \mathbf{L} \xi_{i}^{j}=\mathbf{J} \xi_{i-1}^{j}, \quad j=1,2 .
$$


From inspection of (9), we may see that

$$
\xi_{1}^{j}=\widehat{Z}_{\theta_{j}}, \quad \xi_{2}^{j}=\widehat{Z}_{u_{j}} .
$$

These chains can be continued independently, providing suitable conditions are met, however this turns out to be nongeneric. Surprisingly, the most natural way to extend the lengths of these chains is though a linear combination of the two. The way this arises from the approach taken in this paper is quite natural, and will be demonstrated during the reduction. If one considers the system

$$
\mathbf{L} \xi_{5}=\zeta_{1} \mathbf{J} \widehat{Z}_{u_{1}}+\zeta_{2} \mathbf{J} \widehat{Z}_{u_{2}},
$$

then solvability generates precisely the condition (11). When this criterion is met we generate a chain (albeit in a different basis) of length four, discussed in [26, 27]. This paper takes that one step further, considers the scenario in which the chain is of length six, so that one must consider a additional linear combination. This system is of the form

$$
\mathbf{L} \xi_{7}=\mathbf{J} \xi_{6}-\sum_{i=1}^{2} \kappa_{i} \mathbf{J} \widehat{Z}_{u_{i}} .
$$

Applying solvability generates the system

$$
\mathbf{K}=\mathrm{D}_{\mathbf{u}} \mathbf{B} \boldsymbol{\kappa},
$$

where

$$
\mathbf{K}=\left(\begin{array}{l}
\left\langle\mathbf{J} \widehat{Z}_{\theta_{1}}, \xi_{6}\right\rangle \\
\left\langle\mathbf{J} \widehat{Z}_{\theta_{2}}, \xi_{6}\right\rangle
\end{array}\right), \quad \boldsymbol{\kappa}=\left(\begin{array}{c}
\kappa_{1} \\
\kappa_{2}
\end{array}\right)
$$

It is clear such a system possesses a solution when $\boldsymbol{\zeta}^{T} \mathbf{K}=0$, which for the purposes of this paper we assume to be met. Explicitly, in the context of the stratified shallow water system (2) this amounts to the condition that

$$
a_{11}\left(1-F_{2}^{2}\right)-2 a_{12}+\frac{a_{22}}{r}\left(1-F_{1}^{2}\right)=0 .
$$

This allows for a further two elements, $\xi_{7}, \xi_{8}$, in the chain to exist. Comparing this to the literature (for example, $[8,26]$ ), it is clear that this is precisely when the dispersive term in the $\mathrm{KdV}$ vanishes. Therefore, the degeneracy of the $\mathrm{KdV}$ and the increase in the length of the Jordan chain are directly related. So assuming the chain is length six (so that (11), (17) hold), we may find $\boldsymbol{\kappa}$ as

$$
\boldsymbol{\kappa}=-\frac{u_{2}}{g \rho_{1}(1-r)}\left(r a_{11}+\left(1+r-F_{1}^{2}\right) a_{12}+\left(1-F_{1}^{2}\right) a_{22}\right)\left(\begin{array}{l}
1 \\
0
\end{array}\right)
$$

and define the vector of termination constants

$$
\mathbf{K}_{5}=-\left(\begin{array}{c}
\left\langle\widehat{Z}_{\theta_{1}}, \mathbf{J} \xi_{8}\right\rangle \\
\left\langle\widehat{Z}_{\theta_{2}}, \mathbf{J} \xi_{8}\right\rangle
\end{array}\right) \neq \mathbf{0}
$$


This chain may now be calculated using the definition of $\mathbf{L}$ in (8). However, it becomes clear that only the elements up to $\xi_{6}$ are needed to compute the desired coefficient, since

$$
\begin{aligned}
\sum_{i=1}^{2} \zeta_{i}\left(\mathscr{K}_{5}\right)_{i} & =\sum_{i=1}^{2} \zeta_{i}\left\langle\mathbf{J} \widehat{Z}_{\theta_{i}}, \xi_{8}\right\rangle=\sum_{i=1}^{2} \zeta_{i}\left\langle\widehat{Z}_{u_{i}}, \mathbf{J} \xi_{7}\right\rangle \\
& =-\left\langle\xi_{5}, \mathbf{J} \xi_{6}-\sum_{i=1}^{2} \kappa_{i} \mathbf{J} \widehat{Z}_{u_{i}}\right\rangle=-\left\langle\xi_{5}, \mathbf{J} \xi_{6}\right\rangle-\sum_{i=1}^{2} \kappa_{i}\left\langle\xi_{6}, \mathbf{J} \widehat{Z}_{\theta_{i}}\right\rangle \\
& =\left\langle\mathbf{J} \xi_{5}, \xi_{6}\right\rangle-\boldsymbol{\kappa}^{T} \mathbf{K} .
\end{aligned}
$$

The other elements need only exist abstractly for the asymptotics resulting in the desired PDE. Thus, computing the $\theta_{i}$-independent chain for this problem according to the theory gives the first 4 elements as

$$
\begin{aligned}
& \xi_{1}=(1,0,0,0,0,0,0,0)^{T}, \\
& \xi_{2}=(0,0,1,0,0,0,0,0)^{T}, \\
& \xi_{3}=\frac{1}{g(1-r)}\left(0,-u_{1}, 0, u_{1}, g\left(\rho_{1}-\rho_{2}\right) \eta_{0}-\rho_{1} u_{1}^{2}, 0, \rho_{2} u_{1} u_{2}, 0\right)^{T}, \\
& \xi_{4}=\frac{1}{g(1-r)}\left(0, r u_{2}, 0,-u_{2}, \rho_{2} u_{1} u_{2}, 0, g \rho_{2}(1-r) \chi_{0}-\rho_{2} u_{2}^{2}, 0\right)^{T}, \\
& \xi_{5}=\frac{1}{g(1-r)}\left(0,0,0,0,0,0, a_{11} S_{1}-a_{12} S_{2}, 0, a_{12} S_{1}-a_{22} S_{2}\right)^{T} \\
& \xi_{6}=\frac{1}{g(1-r)}\left(0, T_{1}, 0, T_{2}, \rho_{1} u_{1} T_{1}, 0, \rho_{2} u_{2} T_{2}, 0\right)^{T},
\end{aligned}
$$

with

$$
\begin{aligned}
& S_{1}=\rho_{2} u_{2}\left(g \eta_{0}\left(1-r-F_{1}^{2}\right)+u_{1} u_{2}\right), \quad S_{2}=g \eta_{0} u_{2}\left(\rho_{1}-\rho_{2}\right)\left(1-F_{1}^{2}\right), \\
& T_{1}=\frac{u_{2}}{g\left(\rho_{1}-\rho_{2}\right)}\left(g \rho_{1} \eta_{0}\left(1-r-F_{1}^{2}\right)\left(a_{11} r+(1+r) a_{12}+a_{22}\right)+\rho_{2} u_{1} u_{2}\left(a_{11}+2 a_{12}+a_{22}\right)\right), \\
& T_{2}=-\frac{u_{2}}{g \rho_{2}(1-r)}\left(u_{1} u_{2}\left(a_{11} r+(1+r) a_{12}+a_{22}\right)+g \rho_{1} \eta_{0}\left(1-r-F_{1}^{2}\right)\left(a_{11} r^{2}+2 r a_{12}+a_{22}\right)\right) .
\end{aligned}
$$

These may then be used to find that, after using (12), (13) and (17),

$$
\boldsymbol{\zeta}^{T} \mathbf{K}_{5}=-\rho_{1} \rho_{2} \eta_{0}^{2} \chi_{0}\left(1-r-F_{1}^{2}\right)\left(a_{12}-\frac{a_{22}}{r}\left(1-F_{1}^{2}\right)\right)^{2} .
$$

This will be shown to form the coefficient of highest dispersion in the derived PDE.

\subsubsection{Mixed Chain Analysis}

The analysis also generates a Jordan chain via the mixing of the chain above and one relating to the $\widehat{Z}_{\omega_{i}}$ derivatives, and will be the one which leads to the mixed dispersive term. This is generated from the initial expression

$$
\mathbf{L} \Xi_{1}=\sum_{i=1}^{2}\left[\zeta_{i}\left(\mathbf{M} \widehat{Z}_{u_{i}}+\mathbf{J} \widehat{Z}_{\omega_{i}}\right)-\gamma_{i} \mathbf{J} \widehat{Z}_{u_{i}}\right] .
$$


By imposing solvability, one generates (15) and $\gamma$ can be solved for when (14) holds. This paper assumes that a chain of this form is the minimum length, which is two, and so the existence of $\Xi_{2}$ with

$$
\mathbf{L} \Xi_{2}=\mathbf{M} \xi_{5}+\mathbf{J} \Xi_{1}
$$

and subsequently leads to the condition

$$
\mathbf{T}\left(\begin{array}{c}
\mathscr{T}_{1} \\
\mathscr{T}_{2}
\end{array}\right)=-\left(\begin{array}{c}
\left\langle\widehat{Z}_{\theta_{1}}, \mathbf{M} \xi_{6}+\mathbf{J} \Xi_{2}\right\rangle \\
\left\langle\widehat{Z}_{\theta_{1}}, \mathbf{M} \xi_{6}+\mathbf{J} \Xi_{2}\right\rangle
\end{array}\right) \neq \mathbf{0}
$$

so that the chain terminates after two elements.

In practice, neither of the $\Xi_{i}$ need be calculated to obtain the coefficient of mixed dispersion, but are required to exist abstractly for the asymptotics. This can be seen if one manipulates the projection of $\mathbf{T}$ :

$$
\begin{aligned}
\sum_{i=1}^{2} \zeta_{i} \mathscr{T}_{i} & =-\sum_{i=1}^{2} \zeta_{i}\left\langle\widehat{Z}_{\theta_{i}}, \mathbf{M} \xi_{6}+\mathbf{J} \Xi_{2}\right\rangle=\sum_{i=1}^{2} \zeta_{i}\left(\left\langle\widehat{Z}_{u_{i}}, \mathbf{J} \Xi_{1}+\mathbf{M} \xi_{5}\right\rangle+\left\langle\widehat{Z}_{\omega_{i}}, \mathbf{J} \xi_{5}\right\rangle\right) \\
& =\left\langle\xi_{5}, \sum_{i=1}^{2}\left[\gamma_{i} \mathbf{J} \widehat{Z}_{u_{i}}-2 \zeta_{i}\left(\mathbf{J} \widehat{Z}_{\omega_{i}}+\mathbf{M} \widehat{Z}_{u_{i}}\right)\right]\right\rangle=-\sum_{i=1^{2}}\left[\gamma_{i}\left\langle\xi_{6}, \mathbf{J} \widehat{Z}_{\theta_{i}}\right\rangle+2 \zeta_{i}\left(\mathbf{J} \widehat{Z}_{\omega_{i}}+\mathbf{M} \widehat{Z}_{u_{i}}\right)\right] \\
& =-\gamma \mathbf{K}-2 \sum_{i=1}^{2} \zeta_{i}\left\langle\xi_{5}, \mathbf{J} \widehat{Z}_{\omega_{i}}+\mathbf{M} \widehat{Z}_{u_{i}}\right\rangle .
\end{aligned}
$$

This again subverts calculating the latter elements of the chain and makes the dispersive coefficients easier to obtain. Evaluating this expression, we find

$$
\begin{aligned}
\boldsymbol{\zeta}^{T} \mathbf{T}= & \frac{\rho_{1}^{2} \eta_{0}^{2} \chi_{0}\left(1-r-F_{1}^{2}\right)}{(1-r)}\left(a_{12}-\frac{\left(1-F_{2}^{2}\right)}{r} a_{22}\right) \\
& \times\left(\frac{k_{2}}{\chi_{0}}\left(1-r-(1-2 r) F_{1}^{2}\right)-\frac{k_{1}}{\eta_{0}}\left(1-r-(1-2 r) F_{2}^{2}\right)\right) .
\end{aligned}
$$

\section{Triple Criticality - Can It Be Met?}

The equation we aim to derive requires three conditions to be met simultaneously in order for the reduction to occur. This begs the question - can all three be satisfied concurrently? This section aims to show that such points not only exist, but there is a continuum of such points.

Previously in the literature for background flows with zero velocity, one strategy is to introduce a slowly varying travelling wave co-ordinate $\xi=\varepsilon(x-c t)$ for wavespeed $c$ and small parameter $\varepsilon \ll 1$, as is done in [15,20]. By the introduction of this moving frame of reference, the wavespeed appears in the analysis and may be chosen so that certain linear terms vanish. However this generically means other conditions cannot be satisfied, such as the vanishing of the coefficients of the time derivative, nonlinear or dispersive terms. What will be highlighted in this section, and has been shown in other works [8, 27], is that by considering nontrivial background flows these coefficients may vanish when these velocities 
take certain values. This is the mechanism for which the conditions for (1) to emerge will be met.

There are two approaches one can take here. The first is to consider the intersection of the surfaces each of the conditions (12), (13), (17) defines. There are 8 parameters one can vary (each $u_{i}, \omega_{i}, R_{i}, \rho_{i}$ ) and so in order to visualise these surfaces one must choose 3 to vary and fix the remainder. This is done by fixing the thickness of each layer (which corresponds to fixing the $\left.\omega_{i}, R_{i}\right)$, and then visualising in $\left(u_{1}, u_{2}, r\right)$-space. By varying the thicknesses the surfaces may be manipulated so that all three surfaces intersect, and at such points the equation (1) emerges. An example of this is given in figure 2. In general, the points where all three criterion are met appear in pairs (excluding the point $u_{i}=0$ as this corresponds to $r=1$, a loss of stratification) for each choice of $\eta_{0}, \chi_{0}$, and in general requires the upper layer to be thicker than the lower layer. The limitation to such an approach is that it only provides information for one set of parameter values, and typically does not accurately admit the points of criticality. Despite this, it is much easier to determine when criticality has been met by visualising it in this way, as well as illustrating that criticality is always met in pairs.
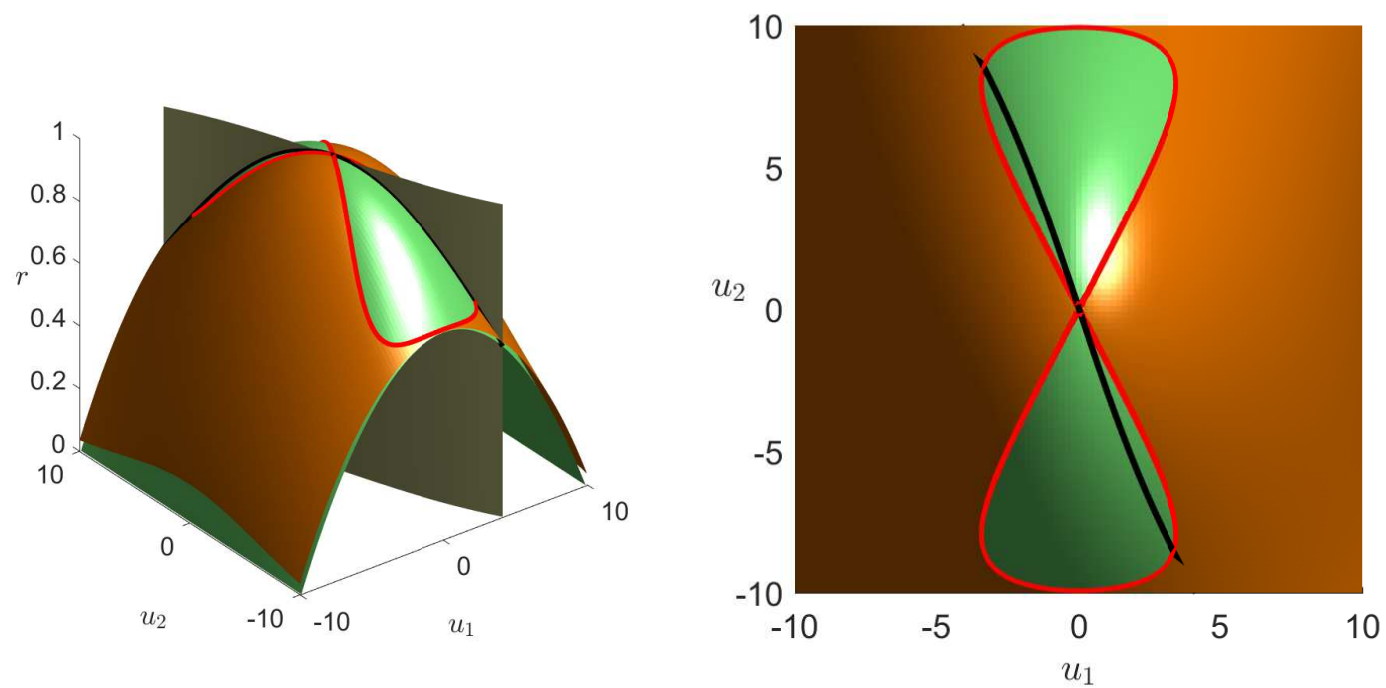

Figure 2: A visualisation of the three conditions (12), (13), (17) which are the green, grey and orange surfaces respectively. The black line shows the intersection between the first two conditions, and the red line the first and third. Where the black and red lines intersect (away from $u_{i}=0$ ) are points where the reduction is obtainable. The above has been computed for $\eta_{0}=10, \chi_{0}=30$.

The second approach is to instead seek such points numerically. As with the surface intersection case, we seek the values in $\left(u_{1}, u_{2}, r\right)$-space where all three conditions are met for each given $\eta_{0}, \chi_{0}$, however in this case the idea will be to solve across a range of given values. By supplying the equations and using a Newton method, one is able to find the 
points where all three conditions simultaneously hold, providing they exist. Moreover, this approach sheds light on the overall trend between $\left(u_{1}, u_{2}, r\right)$ as $\eta_{0}, \chi_{0}$ vary. An example of such a calculation is given in figure 3 , and shows that a large portion of the parameter space admits physical solutions that solve (12), (13), (17) simultaneously, and so such a reduction is quite pervasive.
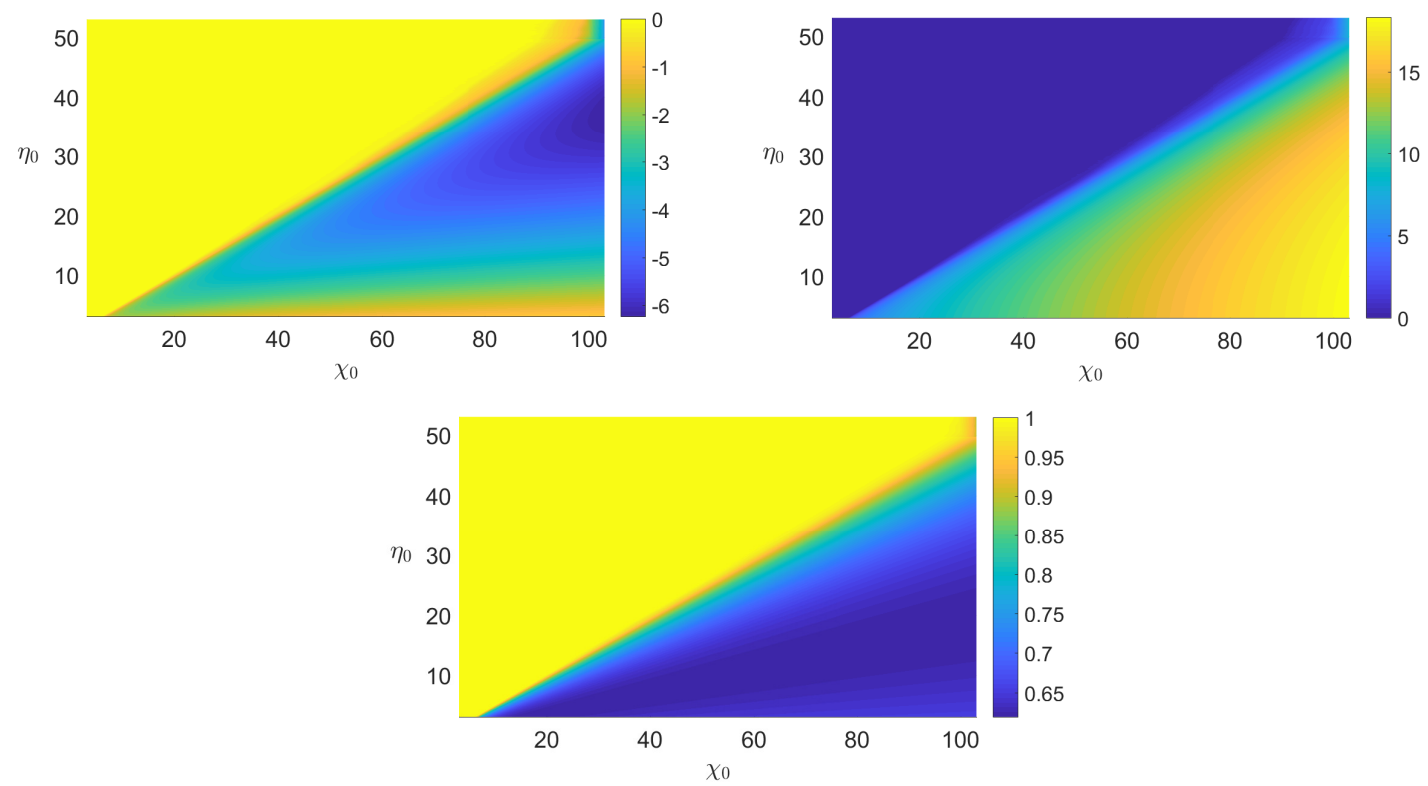

Figure 3: The result of using a Newton scheme to investigate where triple criticality is met, for $u_{1}, u_{2}$ and $r$ respectively. The top left of each image corresponds to the region where $r \approx 1$ and so this are likely inadmissible (as this is close to equivalent densities) The lower right of these (where $r \approx \frac{3}{5}$ ) provides a more physical solution set.

The general trend of the results of the Newton scheme pictured in figure 3 highlight that physically relevant criticalities occur when $\chi_{0} \geq 2 \eta_{0}$. Generically, such a relation does not appear in oceanic systems, but there are specific cases where it may occur. For example, WALLACE AND WILKINSON [29] study the shoaling of periodic waves in a system where $\frac{\chi_{0}}{\eta_{0}} \approx 2.85$ in order to model continental shelves like that of the Eastern Bass Strait. Additional shoaling experiments have been considered by HELFRICH [19] for scenarios with the lower layer being thinner than the top layer. Therefore, it is expected that the equation (1) arises as a relevant model during wave shoaling or as a model for internal waves upon oceanic shelves. Other situations where the equation (1) is suitable are also expected, but are currently less clear.

\section{Summary of the Modulation Reduction}

We now aim to reduce the stratified shallow water system into a single scalar nonlinear PDE using the modulation approach. We use the multisymplectic form of the PDE to do this, since the skew-symmetry of the matrices involved allows for the elimination of several of 
the solvability conditions. Moreover, one only need deal with one set of expressions at each order, rather than explicitly dealing with the four arising from the physical system.

Generically, in order to apply the modulation approach we construct the ansatz

$$
Z=\widehat{Z}\left(\boldsymbol{\theta}+\varepsilon^{3} \boldsymbol{\varphi}(X, T), \mathbf{u}+\varepsilon^{4} \mathbf{q}(X, T), \boldsymbol{\omega}+\varepsilon^{6} \boldsymbol{\Omega}(X, T)\right)+\varepsilon^{5} W(\boldsymbol{\theta}, X, T) .
$$

The slow variables $X, T$ are scaled as

$$
X=\varepsilon x, \quad T=\varepsilon^{3} t,
$$

chosen so that the linear terms in (1) are of the same order, and the modulation functions $\boldsymbol{\varphi}, \mathbf{q}, \boldsymbol{\Omega}$ are related by the conservation of the phase:

$$
\boldsymbol{\theta}_{x}=\mathbf{u}, \quad \boldsymbol{\theta}_{t}=\boldsymbol{\omega} \Rightarrow \boldsymbol{\varphi}_{X}=\mathbf{q}, \quad \boldsymbol{\varphi}_{T}=\boldsymbol{\Omega}, \quad q_{T}=\boldsymbol{\Omega}_{T}
$$

The scalings in (21) have been chosen so that these are of the same order. We also choose to expand $W$, the remainder term used to regulate the analysis, as a simple asymptotic series, so that

$$
W=W_{0}+\varepsilon W_{1}+\varepsilon^{2} W_{2}+\ldots=\sum_{i=0}^{\infty} \varepsilon^{n} W_{n} .
$$

The idea is to then substitute the ansatz (21) into the governing equation (6), Taylor expand around the $\varepsilon=0$ state and solve at each order of $\varepsilon$.

The choice to use this approach is motivated by a few of its advantages. Firstly, by using the ansatz (21) several unimportant terms in the analysis cancel due to properties of the

solution $\widehat{Z}$, which simplifies the analysis throughout. Secondly, it is by using this ansatz and through putting the system into the form (6) that leads to the coefficients having a form related to the conservation laws evaluated along the uniform flow solution $\widehat{Z}$. Finally, due to the generic form of the governing equation (6), the asymptotics need only be done once for a chosen scaling in order to apply to any system that can be put into this form. It is for these reasons that the modulation approach is adopted to obtain the system (27).

However, one may in fact use a postori information to construct a refined ansatz that generates a much simpler analysis. In light of the conditions (11), (14) holding, we may first write

$$
\varphi=\zeta U(X, T)
$$

The function $U$ can be thought of as a scalar velocity potential, related to each flow through the vector $\boldsymbol{\zeta}$, and so $U_{X}$ is a slowly varying velocity perturbation. Secondly, we introduce this and additional terms relating to $\gamma$ and $\boldsymbol{\kappa}$ into the ansatz in the following way:

$$
Z=\widehat{Z}\left(\boldsymbol{\theta}+\varepsilon^{3} \boldsymbol{\zeta} U-\varepsilon^{6} \boldsymbol{\Phi}, \mathbf{u}+\varepsilon^{4} \boldsymbol{\zeta} U_{X}-\varepsilon^{7} \boldsymbol{\Phi}_{X}, \boldsymbol{\omega}+\varepsilon^{6} \boldsymbol{\zeta} U_{T}-\varepsilon^{9} \boldsymbol{\Phi}_{T}\right)+\varepsilon^{5} W(\boldsymbol{\theta}, X, T),
$$

with

$$
\boldsymbol{\Phi}(X, T)=\gamma V(X, T)+\boldsymbol{\kappa} U_{X X}+\boldsymbol{\alpha}(X, T),
$$

where $V$ is a function with the property that

$$
V_{X}=U_{T}
$$


The function $V$ thus has units of length, and so can be thought of as a displacement. The advantage of incorporating these results in advance is three-fold - the first is that there is now only one important unknown function in the analysis, $U$, that will generate the emergent nonlinear PDE. The other, $\boldsymbol{\alpha}$, will be used to ensure the final matrix system is nontrivial and motivate the projection. Secondly is that by assuming the relevant conditions are met, solvability at certain orders of the analysis will happen automatically. Finally, the addition of these terms within the ansatz itself, rather than in $W$, lends itself to the cancellation of several unimportant due to the form of the ansatz, properties of the uniform flow solution and the multisymplectic structure.

This latter ansatz (22) will now be the one substituted into the Euler-Lagrange equations (6). Below is a summary of the results of the reduction, highlighting the key results at each stage.

\section{Leading Order Through To Fifth Order}

One identifies that the leading order recovers (7), and so by construction of the ansatz this is satisfied. In fact, all the orders up to fifth order are also satisfied by construction of the ansatz. Consider, for example, the system at third order, which generates

$$
U\left(\zeta_{1} \mathbf{L} \widehat{Z}_{\theta_{1}}+\zeta_{2} \mathbf{L} \widehat{Z}_{\theta_{2}}\right)=0
$$

which is satisfied by results of the basic state. The terms at fourth order cancel in a similar way but due to results involving the $u_{i}$ derivatives. However, at fifth order one obtains the system

$$
\mathbf{L} W_{0}=U_{X X}\left(\zeta_{1} \mathbf{J} \widehat{Z}_{u_{1}}+\zeta_{2} \mathbf{J} \widehat{Z}_{u_{2}}\right) .
$$

By now considering solvability, one generates the matrix system

$$
\left(\begin{array}{cc}
\left\langle\widehat{Z}_{\theta_{1}}, \mathbf{J} \widehat{Z}_{u_{1}}\right\rangle & \left\langle\widehat{Z}_{\theta_{1}}, \mathbf{J} \widehat{Z}_{u_{2}}\right\rangle \\
\left\langle\widehat{Z}_{\theta_{2}}, \mathbf{J} \widehat{Z}_{u_{1}}\right\rangle & \left\langle\widehat{Z}_{\theta_{2}}, \mathbf{J} \widehat{Z}_{u_{2}}\right\rangle
\end{array}\right) \boldsymbol{\zeta} \equiv-\mathrm{D}_{\mathbf{u}} \mathbf{B} \boldsymbol{\zeta}=\mathbf{0} .
$$

We assume that this is satisfied, and so one may write the solution at this order as

$$
W_{0}=U_{X X} \xi_{5}, \quad \text { with } \quad \mathbf{L} \xi_{5}=\zeta_{1} \mathbf{J} \widehat{Z}_{u_{1}}+\zeta_{2} \mathbf{J} \widehat{Z}_{u_{2}}
$$

\section{Sixth and Seventh Order}

The system at sixth order, once simplified, reads

$$
\mathbf{L} W_{1}=U_{X X X} \mathbf{J} \xi_{5}
$$

This is automatically solvable, since if one considers solvability,

$$
\left\langle\widehat{Z}_{\theta_{i}}, \mathbf{J} \xi_{5}\right\rangle=-\left\langle\widehat{Z}_{u_{i}}, \zeta_{1} \mathbf{J} \widehat{Z}_{u_{1}}+\zeta_{2} \mathbf{J} \widehat{Z}_{u_{2}}\right\rangle \propto\left\langle\widehat{Z}_{u_{1}}, \mathbf{J} \widehat{Z}_{u_{2}}\right\rangle=0
$$

and the last inner product can be shown to vanish explicitly from the uniform flow solution. In this case, the solution at this order is then seen to be

$$
\mathbf{L} W_{1}=U_{X X X} \xi_{6}, \quad \text { with } \quad \mathbf{L} \xi_{6}=\mathbf{J} \xi_{5} .
$$


The seventh order terms can be shown to reduce to

$$
\begin{aligned}
\mathbf{L} W_{2}= & U_{X X X X} \mathbf{J} \xi_{6}-\left(U_{X X X X} \kappa_{1}+V_{X X} \gamma_{1}\right) \mathbf{J} \widehat{Z}_{u_{1}}-\left(U_{X X X X} \kappa_{2}+V_{X X} \gamma_{2}\right) \mathbf{J} \widehat{Z}_{u_{2}} \\
& +\left(\zeta_{1}\left(\mathbf{J} \widehat{Z}_{\omega_{1}}+\mathbf{M} \widehat{Z}_{u_{1}}\right)+\zeta_{2}\left(\mathbf{J} \widehat{Z}_{\omega_{2}}+\mathbf{M} \widehat{Z}_{u_{2}}\right)\right) U_{X T},
\end{aligned}
$$

and when the inner product is taken to assess solvability generates the matrix system

$$
\begin{aligned}
& \left(\begin{array}{ll}
\left\langle\widehat{Z}_{\theta_{1}}, \mathbf{J} \widehat{Z}_{\omega_{1}}+\mathbf{M} \widehat{Z}_{u_{1}}\right\rangle & \left\langle\widehat{Z}_{\theta_{1}}, \mathbf{J} \widehat{Z}_{\omega_{2}}+\mathbf{M} \widehat{Z}_{u_{2}}\right\rangle \\
\left\langle\widehat{Z}_{\theta_{2}}, \mathbf{J} \widehat{Z}_{\omega_{1}}+\mathbf{M} \widehat{Z}_{u_{1}}\right\rangle & \left\langle\widehat{Z}_{\theta_{2}}, \mathbf{J} \widehat{Z}_{\omega_{2}}+\mathbf{M} \widehat{Z}_{u_{2}}\right\rangle
\end{array}\right) \boldsymbol{\zeta} U_{X T}+\left(\begin{array}{l}
\left\langle\widehat{Z}_{\theta_{1}}, \mathbf{J} \xi_{6}\right\rangle \\
\left\langle\widehat{Z}_{\theta_{2}}, \mathbf{J} \xi_{6}\right\rangle
\end{array}\right) U_{X X X X} \\
& -\left(\begin{array}{ll}
\left\langle\widehat{Z}_{\theta_{1}}, \mathbf{J} \widehat{Z}_{u_{1}}\right\rangle & \left\langle\widehat{Z}_{\theta_{1}}, \mathbf{J} \widehat{Z}_{u_{2}}\right\rangle \\
\left\langle\widehat{Z}_{\theta_{2}}, \mathbf{J} \widehat{Z}_{u_{1}}\right\rangle & \left\langle\widehat{Z}_{\theta_{2}}, \mathbf{J} \widehat{Z}_{u_{2}}\right\rangle
\end{array}\right)\left(\boldsymbol{\kappa} U_{X X X X}+\gamma U_{X T}\right)=\mathbf{0}, \\
& \Rightarrow \quad\left(\mathrm{D}_{\mathbf{u}} \mathbf{A}+\mathrm{D}_{\boldsymbol{\omega}} \mathbf{B}\right) \boldsymbol{\zeta} U_{X T}+\mathbf{K} U_{X X X X}=\mathrm{D}_{\mathbf{u}} \mathbf{B}\left(U_{X X X X} \boldsymbol{\kappa}+U_{X T} \boldsymbol{\gamma}\right) .
\end{aligned}
$$

This is satisfied by the assumptions made earlier by the definitions of $\boldsymbol{\gamma}$ and $\boldsymbol{\kappa}$ (15) and (16). Therefore we can solve the equation at this order with

$$
W_{2}=U_{X X X X} \xi_{7}+U_{X T} \Xi_{1}
$$

with

$$
\mathbf{L} \xi_{7}=\mathbf{J} \xi_{6}-\sum_{i=1}^{2} \kappa_{i} \mathbf{J} \widehat{Z}_{u_{i}}, \quad \mathbf{L} \Xi_{1}=\sum_{i=1}^{2}\left(\zeta_{i}\left(\mathbf{J} \widehat{Z}_{\omega_{i}}+\mathbf{M} \widehat{Z}_{u_{i}}\right)-\gamma_{i} \mathbf{J} \widehat{Z}_{u_{i}}\right)
$$

\section{Eighth Order}

The penultimate order gives that

$$
\begin{aligned}
\mathbf{L} W_{3}= & U U_{X X} \sum_{i=1}^{2} \zeta_{i}\left(\sum_{i=j}^{2} \zeta_{j} \mathbf{J} \widehat{Z}_{\theta_{i} u_{j}}-\mathrm{D}^{3} S(\widehat{Z})\left(\widehat{Z}_{\theta_{i}}, \xi_{5}\right)\right) \\
& +U_{X X X X X} \mathbf{J} \xi_{7}+U_{X X T}\left(\mathbf{J} \Xi_{1}+\mathbf{M} \xi_{5}\right) .
\end{aligned}
$$

The $U U_{X X}$ term is solvable, since by differentiating the defining equation for $\xi_{5}$ with respect to $\theta_{i}$ one finds

$$
\mathbf{L}\left(\xi_{5}\right)_{\theta_{i}}=\zeta_{1} \mathbf{J} \widehat{Z}_{\theta_{i} u_{1}}+\zeta_{2} \mathbf{J} \widehat{Z}_{\theta_{i} u_{2}}-\mathrm{D}^{3} S(\widehat{Z})\left(\widehat{Z}_{\theta_{i}}, \xi_{5}\right) .
$$

The last two terms in the above are solvable, since for the first

$$
\left\langle\widehat{Z}_{\theta_{i}}, \mathbf{J} \xi_{7}\right\rangle=-\left\langle\widehat{Z}_{u_{i}}, \mathbf{J} \xi_{6}-\kappa_{1} \mathbf{J} \widehat{Z}_{u_{1}}-\kappa_{2} \mathbf{J} \widehat{Z}_{u_{2}}\right\rangle=\left\langle\xi_{6}, \mathbf{J} \widehat{Z}_{u_{i}}\right\rangle=0,
$$

which can be verified by direct calculation. The other term vanishes since

$$
\begin{aligned}
& \left\langle\widehat{Z}_{\theta_{i}}, \mathbf{J} \Xi_{1}+\mathbf{M} \xi_{5}\right\rangle \\
= & -\left\langle\widehat{Z}_{u_{i}}, \zeta_{1}\left(\mathbf{J} \widehat{Z}_{\omega_{1}}+\mathbf{M} \widehat{Z}_{u_{1}}\right)+\zeta_{2}\left(\mathbf{J} \widehat{Z}_{\omega_{2}}+\mathbf{M} \widehat{Z}_{u_{2}}\right)-\gamma_{1} \mathbf{J} \widehat{Z}_{u_{1}}-\gamma_{2} \mathbf{J} \widehat{Z}_{u_{2}}\right\rangle \\
& -\left\langle\widehat{Z}_{\omega_{i}}, \zeta_{1} \mathbf{J} \widehat{Z}_{u_{1}}+\zeta_{2} \mathbf{J} \widehat{Z}_{u_{2}}\right\rangle \\
= & -\zeta_{1}\left(\left\langle\widehat{Z}_{u_{i}}, \mathbf{J} \widehat{Z}_{\omega_{1}}\right\rangle+\left\langle\widehat{Z}_{\omega_{i}}, \mathbf{J} \widehat{Z}_{u_{i}}\right\rangle+\left\langle\widehat{Z}_{u_{1}}, \mathbf{M} \widehat{Z}_{u_{1}}\right\rangle\right) \\
& +\zeta_{2}\left(\left\langle\widehat{Z}_{u_{i}}, \mathbf{J} \widehat{Z}_{\omega_{2}}\right\rangle+\left\langle\widehat{Z}_{\omega_{i}}, \mathbf{J} \widehat{Z}_{u_{2}}\right\rangle+\left\langle\widehat{Z}_{u_{i}}, \mathbf{M} \widehat{Z}_{u_{2}}\right\rangle\right) \\
\propto & \left\langle\widehat{Z}_{u_{1}}, \mathbf{J} \widehat{Z}_{\omega_{2}}\right\rangle+\left\langle\widehat{Z}_{\omega_{1}}, \mathbf{J} \widehat{Z}_{u_{2}}\right\rangle+\left\langle\widehat{Z}_{u_{1}}, \mathbf{M} \widehat{Z}_{u_{2}}\right\rangle=0,
\end{aligned}
$$


which one can verify by direct calculation using the basic state. Therefore, the solution at this order is

$$
W_{3}=U U_{X X} \sum_{i=1}^{2} \zeta_{i}\left(\xi_{5}\right)_{\theta_{i}}+U_{X X X X X} \xi_{8}+U_{X X T} \Xi_{2}, \quad \mathbf{L} \xi_{8}=\mathbf{J} \xi_{7}, \quad \mathbf{L} \Xi_{2}=\mathbf{J} \Xi_{1}+\mathbf{M} \xi_{5} .
$$

\section{Ninth Order}

The final order of the asymptotics gives the system

$$
\begin{aligned}
\mathbf{L} \widetilde{W}_{4}= & V_{X T} \sum_{i=1}^{2} \gamma_{i}\left(\mathbf{J} \widehat{Z}_{\omega_{i}}+\mathbf{M} \widehat{Z}_{u_{i}}\right)+U_{T T} \sum_{i=1}^{2} \zeta_{i} \mathbf{M} \widehat{Z}_{\omega_{i}}+U_{X X X X X X} \mathbf{J} \xi_{8} \\
& +U_{X} U_{X X} \sum_{i=1}^{2} \zeta_{i}\left(\sum_{j=1}^{2} \zeta_{j} \mathbf{J} \widehat{Z}_{u_{i} u_{j}}+\mathbf{J}\left(\xi_{5}\right)_{\theta_{i}}-\mathrm{D}^{2} S(\widehat{Z})\left(\widehat{Z}_{u_{i}}, \xi_{5}\right)\right) \\
& +U_{X X X T}\left(\mathbf{J} \Xi_{2}+\mathbf{M} \xi_{6}-\sum_{i=1}^{2} \kappa_{i}\left(\mathbf{J} \widehat{Z}_{\omega_{i}}+\mathbf{M} \widehat{Z}_{u_{i}}\right)\right)+\sum_{i=1}^{2}\left(\alpha_{i}\right)_{X X} \mathbf{J} \widehat{Z}_{u_{i}} .
\end{aligned}
$$

The tilde above $W_{4}$ denotes that all terms that are solvable have been absorbed into it, what these are however is not important at this stage. The coefficients generated by the remaining terms are sought instead, and are now calculated.

The first term, in a similar way some of the seventh order terms in (24), gives the tensor term

$$
\left(\mathrm{D}_{\mathbf{u}} \mathbf{A}+\mathrm{D}_{\boldsymbol{\omega}} \mathbf{B}\right) \gamma V_{X T}
$$

The second term gives

$$
\left(\begin{array}{cc}
\left\langle\widehat{Z}_{\theta_{1}}, \mathbf{M} \widehat{Z}_{\omega_{1}}\right\rangle & \left\langle\widehat{Z}_{\theta_{1}}, \mathbf{M} \widehat{Z}_{\omega_{2}}\right\rangle \\
\left\langle\widehat{Z}_{\theta_{2}}, \mathbf{M} \widehat{Z}_{\omega_{1}}\right\rangle & \left\langle\widehat{Z}_{\theta_{2}}, \mathbf{M} \widehat{Z}_{\omega_{2}}\right\rangle
\end{array}\right) \boldsymbol{\zeta} U_{T T} \equiv-\mathrm{D}_{\boldsymbol{\omega}} \mathbf{A} \boldsymbol{\zeta} U_{T T}
$$

The sixth order spatial derivative term generates the expression

$$
\left(\begin{array}{c}
\left\langle\widehat{Z}_{\theta_{1}}, \mathbf{J} \xi_{8}\right\rangle \\
\left\langle\widehat{Z}_{\theta_{2}}, \mathbf{J} \xi_{8}\right\rangle
\end{array}\right) U_{X X X X X X}-\mathbf{K}_{5} U_{X X X X X X}
$$

by earlier definition in (18). The mixed dispersion term, also using earlier working in (24) and the definition (20), gives

$$
\begin{aligned}
& \left(\begin{array}{c}
\left\langle\widehat{Z}_{\theta_{2}}, \mathbf{J} \Xi_{2}+\mathbf{M} \xi_{6}-\sum_{i=1}^{2} \kappa_{i}\left(\mathbf{J} \widehat{Z}_{\omega_{i}}+\mathbf{M} \widehat{Z}_{u_{i}}\right)\right\rangle \\
\left\langle\widehat{Z}_{\theta_{2}}, \mathbf{J} \Xi_{2}+\mathbf{M} \xi_{6}-\sum_{i=1}^{2} \kappa_{i}\left(\mathbf{J} \widehat{Z}_{\omega_{i}}+\mathbf{M} \widehat{Z}_{u_{i}}\right)\right\rangle
\end{array}\right) U_{X X X T} \\
= & {\left[-\left(\begin{array}{c}
\mathscr{T}_{1} \\
\mathscr{T}_{2}
\end{array}\right)+\left(\mathrm{D}_{\mathbf{u}} \mathbf{A}+\mathrm{D}_{\boldsymbol{\omega}} \mathbf{B}\right) \boldsymbol{\kappa}\right] U_{X X X T}=-\left[\mathbf{T}-\left(\mathrm{D}_{\mathbf{u}} \mathbf{A}+\mathrm{D}_{\boldsymbol{\omega}} \mathbf{B}\right) \boldsymbol{\kappa}\right] U_{X X X T} . }
\end{aligned}
$$

The $\alpha$ terms, as seen before in (23), will generate the term

$$
-\mathrm{D}_{\mathbf{u}} \mathbf{B} \boldsymbol{\alpha}_{X X}
$$


The final term to discuss is the nonlinearity. Through manipulation one may show

$$
\begin{aligned}
& \left\langle\widehat{Z}_{\theta_{i}}, \mathrm{D}^{3} S(\widehat{Z})\left(\widehat{Z}_{u_{j}}, \xi_{5}\right)-\mathbf{J}\left(\xi_{5}\right)_{\theta_{j}}-\sum_{k=1}^{2} \zeta_{k} \mathbf{J} \widehat{Z}_{u_{j} u_{k}}\right\rangle \\
& =\left\langle\mathrm{D}^{3} S(\widehat{Z})\left(\widehat{Z}_{u_{j}}, \widehat{Z}_{\theta_{i}}\right)-\mathbf{J} \widehat{Z}_{\theta_{i} \theta_{j}}, \xi_{5}\right\rangle-\sum_{k=1}^{2} \zeta_{k}\left\langle\widehat{Z}_{\theta_{i}}, \mathbf{J} \widehat{Z}_{u_{j} u_{k}}\right\rangle, \\
& =-\left\langle\widehat{Z}_{\theta_{i} u_{j}}, \mathbf{L} \xi_{5}\right\rangle-\sum_{k=1}^{2} \zeta_{k}\left\langle\widehat{Z}_{\theta_{i}}, \mathbf{J} \widehat{Z}_{u_{j} u_{k}}\right\rangle \\
& =-\sum_{k=1}^{2} \zeta_{k}\left(\left\langle\widehat{Z}_{\theta_{i} u_{j}}, \mathbf{J} \widehat{Z}_{u_{k}}\right\rangle+\left\langle\widehat{Z}_{\theta_{i}}, \mathbf{J} \widehat{Z}_{u_{j} u_{k}}\right\rangle\right), \\
& =\sum_{k=1}^{2} \zeta_{k} \partial_{u_{j} u_{k}} \mathscr{B}_{i} .
\end{aligned}
$$

Thus, the tensor acting on the nonlinear term takes the form

$$
-\left(\begin{array}{l}
\zeta_{1}^{2} \partial_{u_{1} u_{1}} \mathscr{B}_{1}+2 \zeta_{1} \zeta_{2} \partial_{u_{1} u_{2}} \mathscr{B}_{1}+\zeta_{2}^{2} \partial_{u_{2} u_{2}} \mathscr{B}_{1} \\
\zeta_{1}^{2} \partial_{u_{1} u_{1}} \mathscr{B}_{2}+2 \zeta_{1} \zeta_{2} \partial_{u_{1} u_{2}} \mathscr{B}_{2}+\zeta_{2}^{2} \partial_{u_{2} u_{2}} \mathscr{B}_{2}
\end{array}\right) U_{X} U_{X X}=-\mathrm{D}_{\mathbf{u}}^{2} \mathbf{B}(\boldsymbol{\zeta}, \boldsymbol{\zeta}) U_{X} U_{X X} .
$$

Therefore, combining all these results and using $V_{X}=U_{T}$ gives the matrix equation

$$
\begin{aligned}
{\left[\mathrm{D}_{\boldsymbol{\omega}} \mathbf{A} \boldsymbol{\zeta}-\left(\mathrm{D}_{\mathbf{u}} \mathbf{A}+\mathrm{D}_{\boldsymbol{\omega}} \mathbf{B}\right) \boldsymbol{\gamma}\right] U_{T T}+\mathrm{D}_{\mathbf{u}}^{2} \mathbf{B}(\boldsymbol{\zeta}, \boldsymbol{\zeta}) U_{X} U_{X X} } \\
+\left[\mathbf{T}-\left(\mathrm{D}_{\mathbf{u}} \mathbf{A}+\mathrm{D}_{\boldsymbol{\omega}} \mathbf{B}\right) \boldsymbol{\kappa}\right] U_{X X X T}+\mathbf{K}_{5} U_{X X X X X X}+\mathrm{D}_{\mathbf{u}} \mathbf{B} \boldsymbol{\alpha}_{X X}=\mathbf{0} .
\end{aligned}
$$

To now obtain the scalar equation desired, we project in the direction of the kernel of $\mathrm{D}_{\mathbf{u}} \mathbf{B}$, $\boldsymbol{\zeta}$ which is achieved by multiplying by this vector on the left. This eliminates the final inhomogeneity and gives

$$
C_{1} U_{T T}+C_{2} U_{X} U_{X X}+C_{3} U_{X X X T}+C_{4} U_{X X X X X X}=0
$$

with

$$
\begin{aligned}
& C_{1}=\boldsymbol{\zeta}^{T}\left[\mathrm{D}_{\boldsymbol{\omega}} \mathbf{A} \boldsymbol{\zeta}-\left(\mathrm{D}_{\mathbf{u}} \mathbf{A}+\mathrm{D}_{\boldsymbol{\omega}} \mathbf{B}\right) \boldsymbol{\gamma}\right]=\boldsymbol{\zeta}^{T} \mathrm{D}_{\boldsymbol{\omega}} \mathbf{A} \boldsymbol{\zeta}-\boldsymbol{\gamma}^{T} \mathrm{D}_{\mathbf{u}} \mathbf{B} \boldsymbol{\gamma} \\
& C_{2}=\boldsymbol{\zeta}^{T} \mathrm{D}_{\mathbf{u}}^{2} \mathbf{B}(\boldsymbol{\zeta}, \boldsymbol{\zeta}) \\
& C_{3}=\boldsymbol{\zeta}^{T}\left[\mathbf{T}-\left(\mathrm{D}_{\mathbf{u}} \mathbf{A}+\mathrm{D}_{\boldsymbol{\omega}} \mathbf{B}\right) \boldsymbol{\kappa}\right]=\boldsymbol{\zeta}^{T} \mathbf{T}-\boldsymbol{\gamma}^{T} \mathrm{D}_{\mathbf{u}} \mathbf{B} \boldsymbol{\kappa} \\
& C_{4}=\boldsymbol{\zeta}^{T} \mathbf{K}_{5}
\end{aligned}
$$

We have the knowledge to compute these explicitly, and so these become

$$
\begin{aligned}
& C_{1}=-g^{2} \rho_{1}^{2} \rho_{2} \eta_{0}^{2} \chi_{0}\left(1-r-F_{1}^{2}\right)\left(\frac{4 u_{1} u_{2}}{g^{2} \eta_{0} \chi_{0}}-\frac{1-F_{1}^{2}}{g \chi_{0}}-\frac{1-F_{2}^{2}}{g \eta_{0}}\right), \\
& C_{2}=3 g^{2} \rho_{1}^{3} \rho_{2} u_{2} \eta_{0}^{2}\left(1-r-F_{1}^{2}\right)\left(\chi_{0} r\left(1-F_{2}^{2}\right) F_{1}^{2}-\eta_{0}\left(1-F_{1}^{2}\right)^{2} F_{2}^{2}\right), \\
& C_{3}=-4 u_{1} \rho_{1}^{2} \eta_{0} \chi_{0}\left(1-r-F_{1}^{2}\right)\left(1-F_{2}^{2}\right)\left(a_{12}-\frac{a_{22}}{r}\left(1-F_{1}^{2}\right)\right) \\
& C_{4}=-\rho_{1} \rho_{2} \eta_{0}^{2} \chi_{0}\left(1-r-F_{1}^{2}\right)\left(a_{12}-\frac{a_{22}}{r}\left(1-F_{1}^{2}\right)\right)^{2}
\end{aligned}
$$


These can be reduced to give

$$
\begin{aligned}
& a_{1}=\left(\frac{4 u_{1} u_{2}}{g^{2} \eta_{0} \chi_{0}}-\frac{1-F_{1}^{2}}{g \chi_{0}}-\frac{1-F_{2}^{2}}{g \eta_{0}}\right), \\
& a_{2}=-\frac{3 \rho_{1} u_{2}}{\chi_{0}}\left(\chi_{0} r\left(1-F_{2}^{2}\right) F_{1}^{2}-\eta_{0}\left(1-F_{1}^{2}\right)^{2} F_{2}^{2}\right), \\
& a_{3}=\frac{4 u_{1}\left(1-F_{2}^{2}\right)}{g^{2} \rho_{2} \eta_{0}}\left(a_{12}-\frac{a_{22}}{r}\left(1-F_{1}^{2}\right)\right) \\
& a_{4}=\frac{1}{g^{2} \rho_{1}}\left(a_{12}-\frac{a_{22}}{r}\left(1-F_{1}^{2}\right)\right)^{2}
\end{aligned}
$$

Finally, differentiate this equation with respect to $X$ and introduce $A=U_{X}$ to obtain the more familiar form of the final PDE,

$$
a_{1} A_{T T}+\left(\frac{1}{2} a_{2} A^{2}+a_{3} A_{X T}+a_{4} A_{X X X X}\right)_{X X}=0 .
$$

This completes the derivation of the higher order Boussinesq equation.

\section{Analysis of the Resulting PDE}

Now that we have derived the desired PDE, we analyse some of its properties and solutions. In particular we investigate the linear dispersion relation (to deduce the stability of trivial states) and study some simple travelling wave solutions for such a system.

We first investigate the linearisation around the trivial state, thus taking $A=\hat{A}$ with $|\hat{A}| \ll 1$ gives the equation

$$
a_{1} \hat{A}_{T T}+\left(a_{3} \hat{A}_{X T}+a_{4} \hat{A}_{X X X X}\right)_{X X}=0 .
$$

Using a normal mode ansatz $\hat{A} \sim e^{i(\alpha x+\beta t)}$ gives that the time exponent $\beta$ satisfies

$$
\beta=\frac{a_{3} \pm \sqrt{a_{3}^{2}-4 a_{1} a_{4}}}{2 a_{1}} \alpha^{3} .
$$

It is therefore quite clear that the emergence of higher order dispersion presents the opportunity for the linear instability of the classical two-way Boussinesq to be stabilised. Moreover, computing the discriminant using (26) reveals that linear stability in this case requires

$$
\frac{4 F_{1}^{2}\left(1-F_{2}^{2}\right)^{2}}{g \rho_{2}^{2} \eta_{0}}-\frac{4}{\rho_{1}}\left(\frac{4 u_{1} u_{2}}{g^{2} \eta_{0} \chi_{0}}-\frac{1-F_{1}^{2}}{g \chi_{0}}-\frac{1-F_{2}^{2}}{g \eta_{0}}\right) \geq 0 .
$$

It is apparent from (13) that $u_{1} u_{2}<0$ and so generically the term in the largest bracket is negative. This means that the zero state is typically linearly stable in this reduction. This is surprising, since when the velocities differ the Kelvin-Helmholtz instability is known to occur [2], however it could be that the emergence of higher order dispersion regularises the instability in an analogous way to surface tension effects. Another possible explanation is that the shallow water model (2) considered here only accounts for third order dispersive

effects, and with higher order dispersion the sign of $a_{4}$ changes and the instability cannot be stabilised. 


\subsection{Numerical Simulation of Travelling Waves}

To investigate travelling wave solutions to (27), we implement a finite difference scheme. For simplicity we consider only the case of a travelling wave co-ordinate $\xi=x-v t$ for wavespeed $v$, so that we solve the ODE

$$
v^{2} a_{1} A^{\prime \prime}+\left(\frac{1}{2} a_{2} A^{2}-v a_{3} A^{\prime \prime}+a_{4} A^{(i v)}\right)^{\prime \prime}=0 .
$$

For the two cases considered in this section, either periodic solutions or solitary waves, the above may be integrated twice to give

$$
a_{4} A^{(i v)}-v a_{3} A^{\prime \prime}+\frac{1}{2} a_{2} A^{2}+v^{2} a_{1} A=Q .
$$

for periodic solutions, where $Q<0$ is some constant. In the case of solutions that decay at infinity, the system can be integrated twice to form instead

$$
a_{4} A^{(i v)}-v a_{3} A^{\prime \prime}+\frac{1}{2} a_{2} A^{2}+v^{2} a_{1} A=0 .
$$

It is the above systems we consider for the numerical scheme. We study the former in the cases where $a_{1} a_{4}<0$, since solitary wave solutions do not appear to exist in such cases, and the latter when $a_{1} a_{4}>0$. Both cases are discussed below.

The finite difference scheme is then employed on the above systems (28) and (29). The initial guess chosen for $(28)$ is

$$
A=-\frac{v^{2}}{a_{2}} \cos \left(\frac{1}{2} \frac{10 \xi}{L}\right)
$$

and for (29) the solitary wave profile

$$
A=-\frac{v^{2}}{a_{2}} \operatorname{sech}^{2}\left(\frac{1}{2} \sqrt{\frac{v^{2} a_{0}}{a_{4}}} \xi\right),
$$

are chosen, the latter for its similarity to the solitary wave solution of the two-way Boussinesq equation. These are selected in order for the scheme to converge to the desired forms of the solution (either periodic or solitary wave) using a Newton method. To simplify matters, we seek symmetric solutions to the above and so $A^{\prime}=A^{\prime \prime \prime}=0$ at $\xi=0$ and one may then solve on the half-line. Additionally in the case of solitary waves we impose that $A=-\frac{v^{2}}{a_{2}}$ at $\xi=0$. Typically the domain is of length $L=100$ with $2^{10}$ to $2^{11}$ gridpoints so that there are at least 10 points per $x$ unit so that the spatial resolution is $0.05 \lesssim \Delta x \lesssim 0.1$. Although this may appear to be a large number of points, the Jacobian resulting from this problem is quintidiagonal and so the scheme computes rather rapidly.

The first set of simulations discussed are for the case $a_{1} a_{4}<0$, so that periodic solutions are sought. This is the case for the equation (27) derived for the shallow water system considered in this paper, as $a_{1}<0$ for all physical choices of $u_{i}, \rho_{i}, \eta_{0}, \chi_{0}$ and clearly $a_{4} \geq 0$ for any parameter choices. Some examples of these simulations are given in figure 4 and 5 , 


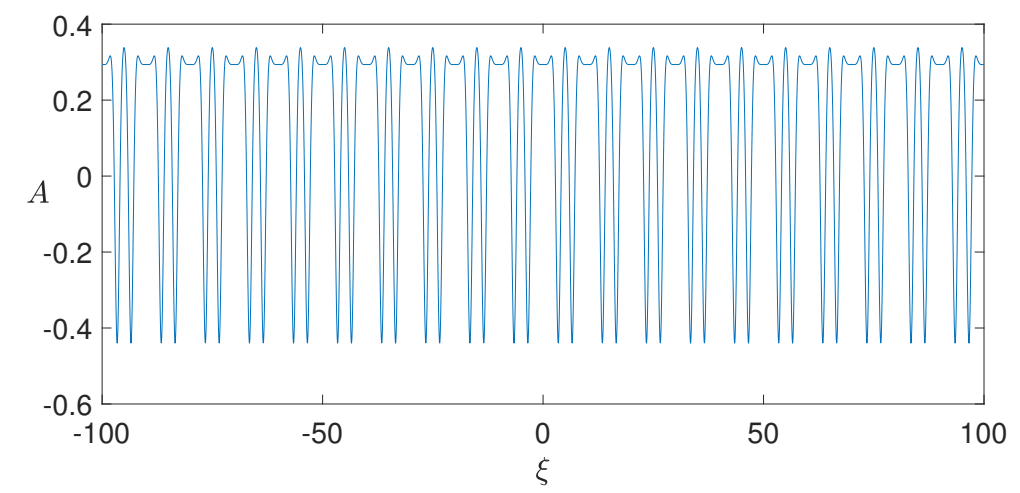

Figure 4: An example of a periodic wave solution to the system (28) with $u_{1}=-1.4781, u_{2}=$ 3.0916, $\rho_{1}=1189, \rho_{2}=1000, \eta_{0}=4, \chi_{0}=8.875$ (4 d.p.), leading to the coefficient values $a_{1}=-0.0389, a_{2}=27.1039 a_{3}=0.0015, a_{4}=0.1008$ (4 d.p.). The parameter $Q$ is chosen to be 2 and the wavespeed $v$ as 5 .

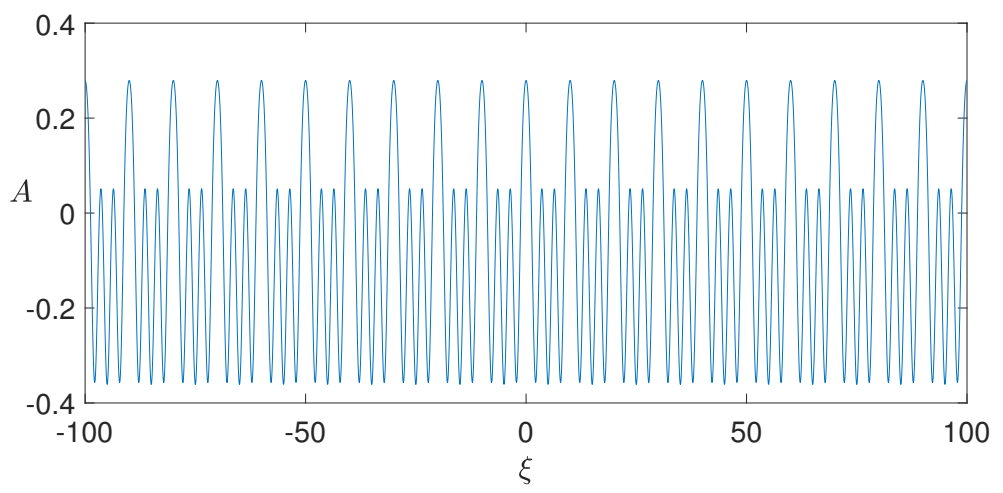

Figure 5: An example of a periodic wave solution to the system (28) with $u_{1}=-1.5219, u_{2}=$ 3.1508, $\rho_{1}=1157 \rho_{2}=1000, \eta_{0}=5, \chi_{0}=10.875$ (4 d.p.), leading to the coefficient values $a_{1}=-0.0311, a_{2}=20.0624 a_{3}=0.0021 a_{4}=0.2889$ ( 4 d.p.). The parameter $Q$ is chosen to be 1 and the wavespeed $v$ as -5 .

depicting various periodic wave profiles and the physical parameters chosen to obtain them. In particular, we note that the numerical scheme highlights the existence of irregular periodic waves, for which the $n^{\text {th }}$ crest is higher than the rest [28]. Other families of periodic waves, such as the classical cnoidal wavetrains, can be found by varying the parameters for the system appropriately.

Another set of interesting simulations arise in cases where $a_{1} a_{4}>0$, which does not arise in the model (27) obtained in this paper for any choice of the parameters. It is however expected to occur when the higher order two-way Boussinesq is derived from the full water wave problem, as fifth order dispersive effects will be taken into account and change the sign of $a_{4}$, as mentioned earlier in this section. Instead, these simulations are done by simply choosing values for the coefficients $a_{i}$. The result of these simulations seems to predict a mixture of classical and generalised solitary waves, as visualised in figures 6 and 7 . The 


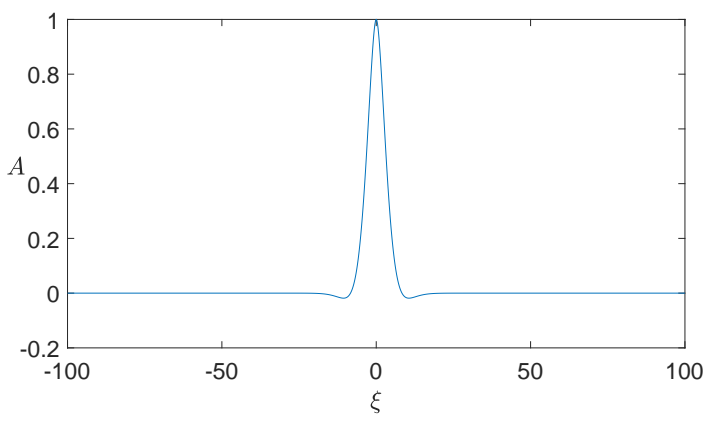

(a)

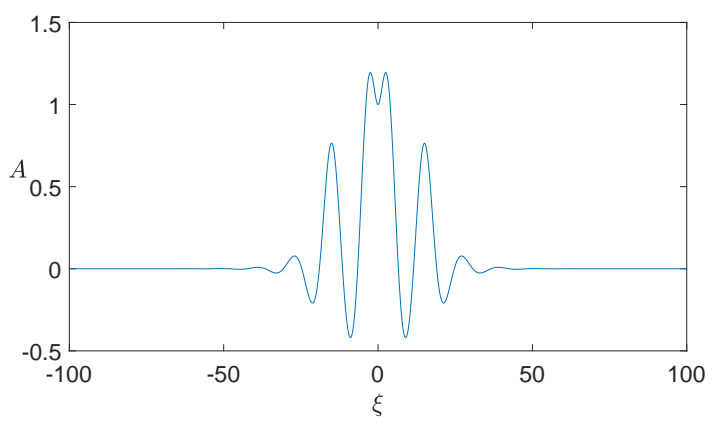

(b)

Figure 6: An example of a solitary wave solution to (29) with $v=-1, a_{1}=1, a_{2}=-2, a_{4}=$ 10 and $(6 \mathrm{a}) a_{2}=-5(6 \mathrm{~b}) a_{2}=5$. Figure (6a) is reminiscent of a classical single hump solitary wave, whereas (6b) demonstrates how (29) admits generalised solitary wave packets.

more classical results appear to occur in cases where

$$
v a_{3}>0
$$

and have similar form to those appearing from fifth order KdV models (for example, [18]). When the opposite is true, the central peak is surrounded by smaller ones (with similar solitary waves appearing in [23]). The width of the range in which these peaks occur increases as $v a_{3}$ decreases. This is akin to homoclinic snaking $[9,10]$, and so the generalised solitary wave found can be viewed as localised patterns to the ODE (29). This is expected, as the structure of (29) and the Swift-Hohenberg are very similar.

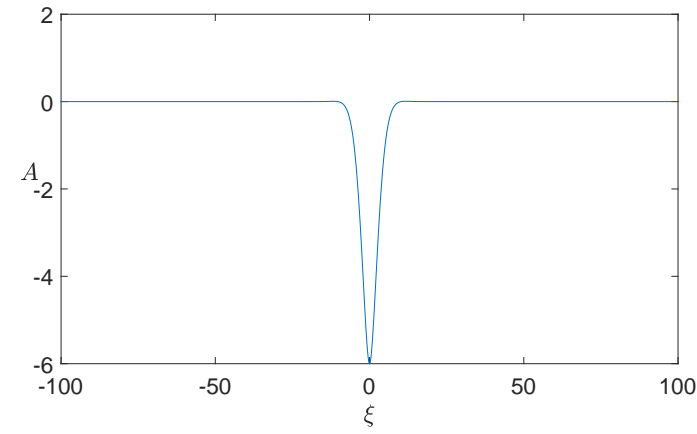

(a)

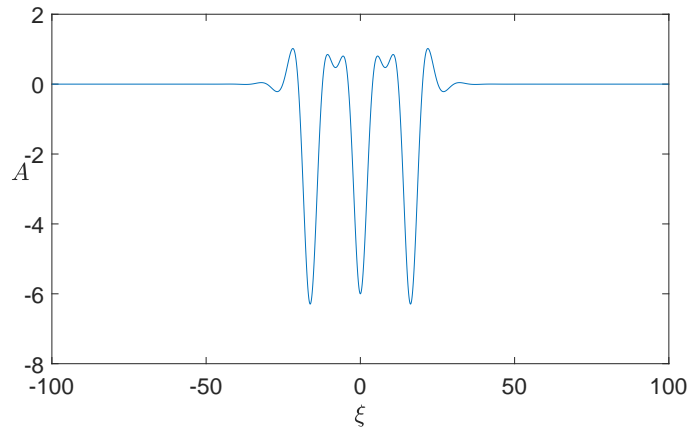

(b)

Figure 7: An example of a solitary wave solution to (29) with $v=2, a_{1}=1, a_{1}=\frac{4}{3}, a_{3}=$ $5, a_{4}=18$ and (7a) $a_{2}=-5(7 \mathrm{~b}) a_{2}=5$. 


\section{Concluding Remarks}

It has now been demonstrated that the stratified shallow water equations may reduce to a highly dispersive two-way nonlinear wave equation. Additionally, it was shown that the regimes in which this system is attainable form a large continuum. Finally, the presence of generalised solitary waves within this model, likely owing to the higher dispersive effects, has been highlighted using numerical methods.

The natural extension to this work is to investigate the full stratified water wave problem in a similar way in order to see if a similar model emerges. The method presented here must be modified somewhat, since the full water wave problem does not possess constant skew symmetric operators $\mathbf{M}$ and $\mathbf{J}$, instead these are functions of the state variable $Z$ which alters the reduction procedure. The approach to do so is expected to generalise the calculations of Bridges for the single layer Euler equations [5] to stratified flows, with the appropriate changes. Additionally, studying the full stratified water wave problem would answer the question posed in section $\S 5$ regarding the omission of higher order dispersive effects from (2) and the linear stability of the model (27).

The large parameter space of this system is likely to support a large number of reduced systems. This has already been seen in the modified KdV obtained by Grimshaw [17] and the higher order KdV attained by Koop and Butler [20] from stratified systems, and so one expects cases where the nonlinearity is enhanced in both the one-way and two-way propagation systems.

This present work has focussed on the case of two layered stratification, which can be generalised to systems with three or more layers. In such environments there is the potential for coupled systems to occur (as has been seen in $[8,16]$ ), which can then have their dispersive properties and nonlinearity enhanced through criticality. A similar study to that undertaken here in such cases is expected to lead to the coupled $\mathrm{KdV}$, with the potential for other coupled systems to occur when suitable conditions are met

The abstract representation of the reduction, although here it focussed on the stratified shallow water system, suggests that this result applies for any system with a two-phase basic state that can be put into the form (6) so long as all the relevant conditions can be met. For example, the coupled Nonlinear Schrodinger equation discussed in [27] may admit the system (27) when the dispersive term in the Boussinesq equation derived therein vanishes.

The numerical study of the derived system here has only focussed on solitary wave solutions, however more exotic solutions may exist. This is suggested by considering the size of the system's phase space (which is 4 dimensional). A starting point for this may be to look at periodic solutions of this system and assess how these differ from those which appear in other models, such as the KdV and two-way Boussinesq equations. Additionally homoclinic snaking could be further explored, and its relevance within the two-layered hydrodynamical system assessed.

\section{Acknowledgements}

The author would like to thank Prof. Tom Bridges for his discussions and insight during the formulation of this work. The author is in receipt of a fully funded Ph.D studentship under 
the EPSRC grant EP/L505092/1.

\section{References}

[1] P.G. Baines, A general method for determining upstream effects in stratified flow of finite depth over long two-dimensional obstacles, J. Fluid Mech. 188, 1-22 (1988).

[2] T.B. Benjamin and T.J. Bridges, Reappraisal of the Kelvin-Helmholtz problem. Part 1. Hamiltonian structure, J. Fluid Mech. 333, 301-325 (1997).

[3] G.S. Benton, The occurence of critical flow and hydraulic jumps in a multi-layered fluid system, J. Meteor. 11, 139-150 (1954).

[4] T.J. Bridges, A universal form for the emergence of the Korteweg-de Vries equation, Proc. Roy. Soc. Lond. A 469, 20120707 (2013).

[5] T.J. Bridges, Symmetry, Phase Modulation and Nonlinear Waves, Cambridge University Press (2017).

[6] T.J. Bridges and N.M. Donaldson, Criticality manifolds and their role in the generation of solitary waves for two-layer flow with a free surface, Euro. J. Mech. B/Fluids 28, 117-126 (2007).

[7] T.J. Bridges \& F.E. Laine-Pearson, Multisymplectic relative equilibria, multiphase wavetrains, and coupled nls equations, Stud. Appl. Math. 107, 137-155 (2001).

[8] T.J. Bridges and D.J. Ratliff, Double criticality and the two-way Boussinesq equation in stratified shallow water hydrodynamics, Phys. Fluids 28062103 (2016).

[9] J. Burke and E. Knobloch, Localized states in the generalized Swift-Hohenberg equation, Phys. Rev. E, 73, 056211 (2006).

[10] J. Burke and E. Knobloch, Homoclinic snaking: Structure and stability, Chaos 17 037102 (2007).

[11] W. Craig, P. Guyenne, and H. Kalisch, Hamiltonian long-wave expansions for free surfaces and interfaces, Commun. Pure Appl. Math., 58.12, 1587-1641 (2005).

[12] W. Craig, P. Guyenne, and C. Sulem, Coupling between internal and surface waves, Nat. Hazards, 57.3, 617-642 (2011).

[13] P. Daripa, Higher-order Boussinesq equations for two-way propagation of shallow water waves, Eur. J. of Mech. B Fluids, 25.6, 1008-1021 (2006).

[14] N.M. Donaldson, Criticality theory and conformal mapping techniques for single and two layer water-wave systems, Ph.D. thesis, University of Surrey, (2006). 
[15] V.D. Djordjevic and L. G. Redekopp, The fission and disintegration of internal solitary waves moving over two-dimensional topography, J. Phys. Oceanogr., 8.6, 1016-1024 (1978).

[16] R. Grimshaw, Models for long-wave instability due to a resonance between two waves, Trends in Application of Mathematics to Mechanics 183-192 (1999).

[17] R.Grimshaw, E. Pelinovsky and T. Talipova, The modified Korteweg-de Vries equation in the theory of large-amplitude internal waves, Nonlinear Proc. Geoph. 4.4, 237-250 (1997).

[18] P. Guyenne and E. I. Parau, Asymptotic modeling and numerical simulation of solitary waves in a floating ice sheet, The Twenty-fifth International Ocean and Polar Engineering Conference, International Society of Offshore and Polar Engineers, (2015).

[19] K.R. Helfrich, Internal solitary wave breaking and run-up on a uniform slope, J. Fluid Mech. 243, 133-154 (1992).

[20] C.G. Koop and, and G. Butler, An investigation of internal solitary waves in a two-fluid system, J. Fluid Mech., 112, 225-251 (1981).

[21] F.E. Laine-Pearson and T.J. Bridges, Nonlinear counterpropagating waves, multisymplectic geometry, and the instability of standing waves, SIAM J. Appl. Math. 64, 20962120 (2004).

[22] G.A. Lawrence, On the hydraulics of Boussinesq and non-Boussinesq two-layer flows, J. Fluid Mech. 215, 457-480 (1990).

[23] B. Malomed and J. M. Vanden-Broeck, Solitary wave interactions for the fifth-order KdV equation Contemporary Mathematics 200 (1996) 133-144.

[24] D.J. Ratliff and T.J. Bridges, Phase dynamics of periodic waves leading to the Kadomtsev-Petviashvili equation in $3+1$ dimensions, Proc. R. Soc. Lond. A 471, 20150137 (2015).

[25] D.J. Ratliff \& T.J. Bridges, Whitham modulation equations, coalescing characteristics, and dispersive Boussinesq dynamics, Physica D, 333, 107-116 ( 2016).

[26] D.J. Ratliff \& T.J. Bridges, Multiphase wavetrains, singular wave interactions, and the emergence of the KdV equation, Proc. R. Soc. Lond. A 472, (2016).

[27] D.J. Ratliff, Double Degeneracy of Multiphase Modulation and the Emergence of the Two-Way Boussinesq Equation, Stud. Appl. Math. (in press), (2017).

[28] J.M. Vanden-Broeck, New families of pure gravity waves in water of infinite depth, Wave Motion 72, 133-141 (2017) .

[29] B.C. Wallace, \& D. L. Wilkinson, Run-up of internal waves on a gentle slope in a two-layered system, J. Fluid Mech. 191, 419-442 (1988). 